\title{
Ausbildungsmotive und die Zeitaufteilung der Auszubildenden im Betrieb
}

Felix Wenzelmann

Angenommen: 8. Februar 2012 / Online publiziert: 6. März 2012

(C) Institut für Arbeitsmarkt- und Berufsforschung 2012

Zusammenfassung Der vorliegende Beitrag untersucht auf Basis der Erhebung zu Kosten und Nutzen der betrieblichen Ausbildung des Bundesinstituts für Berufsbildung (BIBB) den Zusammenhang zwischen dem Ausbildungsmotiv des Ausbildungsbetriebs und der Aufteilung der Arbeitszeit der Auszubildenden im Betrieb. Unterschieden wird zwischen dem Investitions- und dem Produktionsmotiv. Die Zuordnung erfolgt anhand der Übernahmestrategie der Betriebe, die eine generelle, eine teilweise oder keine (bzw. nur in Ausnahmefällen) Weiterbeschäftigung der Auszubildenden nach Abschluss der Ausbildung vorsehen kann. Bei der Zeitaufteilung der Auszubildenden wird unterschieden zwischen Zeiten, in denen einfache Tätigkeiten ausgeführt werden, Zeiten für Fachkräftetätigkeiten und sonstigen ,unproduktiven“Zeiten, in denen die Auszubildenden auf verschiedene Weise ausgebildet werden. Es zeigt sich, dass es in den Betrieben, die dem Investitionsmotiv zugeordnet werden, einen höheren Anteil an ,unproduktiven“ Tätigkeiten als in den produktionsorientierten Betrieben gibt. Darüber hinaus weisen auch weitere Faktoren einen Zusammenhang mit der Zeitaufteilung auf. So kann insbesondere ein positiver Zusammenhang der vor der Ausbildung erworbenen, allgemeinen schulischen Kompetenzen der Auszubildenden mit den Einsatzzeiten für Fachkräftetätigkeiten festgestellt werden.

Schlüsselwörter Betriebliche Ausbildung ·

Produktionsmotiv · Investitionsmotiv ·

Ausbildungsorganisation · Humankapital

F. Wenzelmann $(\bowtie)$

Kosten, Nutzen, Finanzierung, Bundesinstitut für Berufsbildung (BIBB), Robert-Schuman-Platz 3, 53175 Bonn, Deutschland e-mail:wenzelmann@bibb.de
JEL Klassifikationen D22 · J24 · J31

Training motives and the allocation of productive tasks in apprenticeship training in Germany

Abstract This Paper analyzes training motives of firms and the allocation of productive tasks at the workplace using data from the cost-benefit-survey of the Federal Institute for Vocational Education and Training for the year 2007 (BIBB-CBS 2007). We distinguish between two training motives, the investment and the production motive of training. The identification of these motives is via the retention strategy of the firm, which can be: retaining all apprentices, retaining only a part of them or retaining only in exceptional cases.

The time apprentices are available for the firm is divided up into three categories: times of unskilled productive tasks, times of skilled productive tasks and times of "unproductive" tasks. It can be shown that in investment oriented firms the shares of unproductive tasks are higher than in firms following the production motive. Additionally we analyze which other factors influence the allocation of task in the training firm. Especially the general school competence the apprentices have achieved before starting the training has a positive influence on the share of skilled tasks.

\section{Einleitung}

Betriebe haben bezüglich der eigenen Ausbildung von Jugendlichen drei grundlegende Fragen zu beantworten. Erstens müssen sie entscheiden, ob und wenn ja in welchem Umfang sie ausbilden möchten und ggfs. die dafür erforderlichen Voraussetzungen schaffen. Falls die Betriebe sich für eine Ausbildung entscheiden, ist zweitens zu überlegen, 
ob die Weiterbeschäftigung der Auszubildenden nach dem Ausbildungsabschluss angestrebt wird. Und drittens ist $\mathrm{zu}$ entscheiden, wie die Ausbildung in ihrem Betrieb organisiert werden kann.

Die drei Fragen stehen in Wechselbeziehungen zueinander: So lässt sich die erste Frage - jedenfalls rational - nur beantworten, wenn Überlegungen zu den anderen Fragen angestellt wurden und der Betrieb sich eine Erwartung hinsichtlich der entstehenden Kosten, aber auch des Nutzens bilden konnte. ${ }^{1}$ Außerdem kann vermutet werden, dass die Organisation der Ausbildung durch das Übernahmeinteresse beeinflusst wird, da die Organisation zum einen die Qualität der Ausbildung und damit die spätere Einsetzbarkeit als Fachkräfte bestimmt und zum anderen die unmittelbaren Kosten und Erträge der Ausbildung von dieser abhängen.

Die Hypothese der Wechselbeziehung zwischen Ausbildungsmotiv und Ausbildungsorganisation steht im Zentrum dieses Artikels. ${ }^{2}$ Zusätzlich wird untersucht, welche weiteren Faktoren die Organisation der Ausbildung beeinflussen. Ein bedeutender Faktor sind dabei die Auszubildenden selbst. Ihre Fähigkeiten und Kenntnisse beeinflussen die Einsetzbarkeit im Produktionsprozess auf der einen Seite und den Umfang der notwendigen arbeitsplatzfernen Ausbildungsmaßnahmen auf der anderen Seite. Eine Approximation dieser Kenntnisse und Fähigkeiten bieten die allgemeinen schulischen Kompetenzen der Auszubildenden. Der Einsatz der Auszubildenden im Betrieb wird aber auch durch verschiedene exogene Faktoren beeinflusst. So sind in den Ausbildungsordnungen der Berufe Vorgaben enthalten, welche Ausbildungsinhalte im Verlauf der Ausbildung im Betrieb zu vermitteln sind. Trotz dieser institutionellen Einschränkungen bleibt den Betrieben ein relativ großer Spielraum bei der Organisation der Ausbildung vor Ort. Auch Betriebsmerkmale, wie beispielsweise die Betriebsgröße, können einen Einfluss auf den möglichen Arbeitseinsatz der Auszubildenden haben. So wechseln die Auszubildenden in Großbetrieben häufiger zwischen den Abteilungen, um einen Überblick über den gesamten Betrieb zu bekommen. In jeder Abteilung sind dann zunächst Phasen nötig in denen die Auszubildenden diese kennenlernen und daher nicht produktiv eingesetzt werden können.

Die Organisation der Ausbildung wird über die Verteilung der Zeit der Auszubildenden im Betrieb operationalisiert. Die Auszubildenden können ihre Zeit im Betrieb

\footnotetext{
${ }^{1}$ Für eine ausführliche Darstellung und Diskussion der Kosten- und Nutzenebenen der betrieblichen Ausbildung vgl. Schönfeld et al. (2010).

${ }^{2}$ Da nur ausbildende Betriebe betrachtet werden können, kann die Frage nach der generellen Ausbildungsentscheidung nicht empirisch untersucht werden.
}

mit einfachen oder Fachkräftetätigkeiten verbringen oder sie werden auf verschiedene Art und Weise unproduktiv ausgebildet. Unproduktiv meint dabei, dass für den Betrieb unmittelbar keine verwertbaren Produkte oder Dienstleistungen entstehen.

Das Ausbildungsmotiv der Betriebe wird über die Übernahmestrategie operationalisiert. Betrieben, die ,auf jeden Fall weiterbeschäftigen" wollen, wird ein Investitionsmotiv zugeschrieben (vgl. Merrilees 1983), da der zentrale Nutzen der Ausbildung die Sicherung des eigenen Fachkräftebedarfs ist und die Ausbildungsphase als Investition gesehen werden kann. Betrieben, die keine Übernahme der Auszubildenden planen, da sie entweder keinen Fachkräftebedarf erwarten oder diesen über andere Wege decken, wird ein Produktionsmotiv (vgl. Lindley 1975) zugeschrieben. Rational handelnde Ausbildungsbetriebe mit diesem Motiv müssen versuchen, die anfallenden Ausbildungskosten während der Ausbildung durch die Beiträge der Auszubildenden zum Betriebsoutput zu decken, da ein Nutzen nach Ausbildungsende ausgeschlossen ist.

Der vorliegende Beitrag ergänzt die vorhandene empirische Literatur zu den Ausbildungsmotiven in zwei Punkten. Zum einen kann auf den aktuellen Datensatz der BIBBKosten-Nutzen-Erhebung 2007 (BIBB-CBS 2007) (vgl. Schönfeld et al. 2010) zurückgegriffen werden, der gegenüber anderen Datenquellen den Vorteil hat sich explizit auf Fragen der Ausbildungsorganisation im Betrieb zu beziehen. Zum anderen wird erstmals das Motiv über eine direkt erfragte strategische Ausrichtung bezüglich der Übernahme modelliert. Dies hat gegenüber der tatsächlichen Übernahmequote den Vorteil, dass externe Faktoren wie eine veränderte wirtschaftliche Situation, das freiwillige Ausscheiden oder das Poaching ${ }^{3}$ von Absolventen keinen verzerrenden Einfluss haben können.

Es ist zu beachten, dass u.a. aufgrund der Eigenschaften des Querschnittdatensatzes keine kausalen Aussagen aus den Ergebnissen der durchgeführten Regressionsanalysen abgeleitet werden können. Die gefundenen Koeffizienten liefern dennoch interessante Erkenntnisse bezüglich der untersuchten Zusammenhänge und können als Ausgangspunkte für eine inhaltliche Diskussion verwendet werden.

Der Artikel gliedert sich wie folgt auf: Im zweiten Abschnitt werden, ausgehend von der Literatur, Hypothesen entwickelt. Im dritten Abschnitt wird der Datensatz kurz vorgestellt, im vierten Abschnitt werden die Variablen erläutert, mit denen dann die Hypothesen empirisch überprüft werden. Abschnitt fünf fasst zusammen und zieht Schlussfolgerungen.

\footnotetext{
${ }^{3}$ Mohrenweiser et al. (2011) zeigen, dass im dualen System nur wenige Betriebe „Opfer“ von Poaching werden.
} 


\section{Theorie und Hypothesen}

In diesem Abschnitt werden die theoretischen und empirischen Arbeiten zu den Ausbildungsmotiven und dem Einsatz der Auszubildenden im Ausbildungsbetrieb diskutiert. Ausgehend von dieser Literatur werden die zentralen Hypothesen des Artikels hergeleitet.

Nach Becker (1964) sind Betriebe nur dann zu Investitionen in das Humankapital ihrer Mitarbeiter bereit, wenn sie betriebsspezifischer Natur sind. Betriebsspezifisches Humankapital erhöht die Produktivität nur in dem Betrieb, in dem die Fähigkeiten oder das Wissen erworben wurden. Aus diesem Grund kann der Betrieb nach der Ausbildungsphase einen Lohn unterhalb der Produktivität zahlen ohne Gefahr zu laufen, dass der Mitarbeiter den Betrieb verlässt und die Investition somit verloren wäre. Üblicherweise wird jedoch davon ausgegangen, dass bei der dualen Ausbildung allgemeines Humankapital generiert wird (vgl. z.B. Acemoglu und Pischke 1999a), das durch die zertifizierte Abschlussprüfung für alle Marktteilnehmer erkennbar ist. Über die Ausbildungsordnungen werden Mindestmaße an zu erlernenden Wissen und Fähigkeiten festgelegt. ${ }^{4}$ Auf Basis dieser Erkenntnis und der Ergebnisse verschiedener Kosten-Nutzen-Erhebungen, ${ }^{5}$ dass den ausbildenden Betrieben zunächst Kosten durch die Ausbildung entstehen (Bruttokosten ${ }^{6}$ ), stellt sich die Frage, warum Betriebe sich an der dualen Ausbildung beteiligen und wie sie die Ausbildung organisieren, um die entstehenden Kosten zu kompensieren. Dabei wird in der Regel zwischen den beiden Ausbildungsmotiven „Produktionsmotiv“"7 und ,Investitionsmotiv" unterschieden.

Betriebe, die dem Produktionsmotiv folgen, decken die anfallenden (Brutto-)Kosten der Ausbildung bereits während der Ausbildungszeit durch die produktiven Tätigkeiten der Auszubildenden (Erträge). Daher haben diese Betriebe in der Regel kein oder nur ein geringes Interesse an der Übernahme der fertig ausgebildeten Jugendlichen. Rentabel ist der produktive Einsatz der Auszubildenden insbesondere dann, wenn die Lohnstückkosten geringer sind als die von Ungelernten oder Fachkräften (vgl. Mohrenweiser und Backes-Gellner 2010). Im Gegensatz zu den produktionsorientierten Betrieben ist für Betriebe mit Investitionsmotiv

\footnotetext{
${ }^{4}$ Pfeifer et al. (2011) zeigen, dass dennoch in geringem Umfang betriebsspezifisches Humankapital in der dualen Ausbildung generiert wird.

${ }^{5}$ Vgl. z.B. von Bardeleben et al. (1995), Beicht et al. (2004) oder Schönfeld et al. (2010).

${ }^{6}$ Die Begriffe Bruttokosten, Erträge und Nettokosten werde nach den Definitionen von Schönfeld et al. (2010) verwendet, wobei die Nettokosten die Differenz aus Bruttokosten und Erträgen sind.

${ }^{7}$ In einigen Publikationen wird anstelle des Begriffs „Produktionsmotiv“ der Begriff „Substitutionsmotiv“ verwendet, der sich darauf bezieht, dass die Auszubildenden ein Substitut für „normale“ Arbeitskräfte darstellen (vgl. z.B. Mohrenweiser und Backes-Gellner 2010).
}

das zentrale Ziel der Ausbildung die Deckung des zukünftigen Fachkräftebedarfs. Kosten, die während der Ausbildung entstehen, werden in Kauf genommen, da dem Betrieb durch die Übernahme ein Nutzen entsteht, der die Ausbildungsinvestition kompensiert. Es ist jedoch nicht ausgeschlossen, dass auch bei solchen Betrieben die Ausbildungskosten bereits während der Ausbildungsphase gedeckt werden und sie somit einen ,doppelten“ Nutzen erzielen können.

Nutzen durch Übernahme entsteht zum einen durch die Einsparung von Rekrutierungskosten, die bei der Personalgewinnung über den Arbeitsmarkt anfallen. Zum anderen kann der Betrieb aufgrund von Marktunvollkommenheiten (z.B. Mobilitätskosten oder asymmetrische Informationen) den selbst Ausgebildeten einen Lohn unterhalb ihrer Produktivität zahlen und somit einen zusätzlichen Nutzen generieren. Zusätzlich führt eine komprimierte Lohnstruktur dazu, dass der Lohn in geringerem Umfang als die Produktivität der Mitarbeiter steigt. Mit steigender Qualifikation nimmt also die Rente des Betriebs zu. Ausbildungsinvestitionen können mit dieser höheren Rente refinanziert werden. Ursächlich für eine komprimierte Lohnstruktur sind z.B. Tarif- oder Mindestlöhne, die institutionell gesetzt werden und für geringere Lohndifferenzen zwischen den Qualifikationsgruppen sorgen sollen. ${ }^{8}$ Kessler und Lülfesmann (2006) zeigen, dass für Betriebe eine Investition in allgemeines Humankapital sinnvoll sein kann, wenn gleichzeitig auch in spezifisches investiert wird. Dies gilt sogar auch dann, wenn die beiden Humankapitalarten keine Komplemente sind. Der Investitionsanreiz für die Betriebe besteht, da sie im Anschluss an die Investition Löhne unterhalb der Produktivität im eigenen Betrieb zahlen können. Sofern die Löhne oberhalb des alternativ am Arbeitsmarkt erzielbaren Lohns liegen, muss ein Arbeitsplatzwechsel, der „SunkCosts“ für den Betrieb zur Folge hätte, nicht befürchtet werden.

Neben den beiden vorgestellten Kernmotiven für betriebliche Ausbildung werden in der Literatur das Reputationsmotiv (vgl. Sadowski 1980) und das Motiv, die Ausbildung als Screening-Instrument zu verwenden (vgl. Stevens 1994 oder Franz und Zimmermann 2002), genannt. Beim Reputationsmotiv bildet der Betrieb aus, um sein Ansehen (Image) bei Kunden, Zulieferern und Mitarbeitern zu verbessern. Als Screening kann die Ausbildung gesehen werden, wenn der Betrieb die Ausbildung als verlängerte Probezeit einsetzt, um die am besten passenden Mitarbeiter zu rekrutieren. Häufig ist das Motiv eines Ausbildungsbetriebs aber nicht eindimensional, so können sowohl eher investitionsorientierte als auch eher produktionsorientierte Betriebe eine zusätzliche Motivation aus der Steigerung der Reputation oder durch das Screening ziehen.

\footnotetext{
${ }^{8}$ Vgl. Acemoglu und Pischke (1998, 1999a, 1999b) oder Wolter und Ryan (2011) für eine Überblick über die Literatur zu diesem Themengebiet.
} 
Verschiedene empirische Artikel befassen sich mit dem Investitions- und Produktionsmotiv der dualen Ausbildung in Deutschland. Dietrich (2008) untersucht den Einfluss des Ausbildungsmotivs des Betriebs auf den Beschäftigungserfolg der Auszubildenden. Auf Basis der vom BIBB für das Jahr 2000 ermittelten, auf Berufsebene aggregierten Nettokosten (vgl. Beicht et al. 2004) analysiert er den Erfolg der Absolventen der einzelnen Berufe auf dem Arbeitsmarkt. Die Berufe stuft er dabei nach der Höhe der durchschnittlichen Nettokosten der Ausbildung in drei Kategorien ein. Zusätzlich zu den beiden Strategien, die ausschließlich durch das Investitions- bzw. das Produktionsmotiv bestimmt sind, definiert er eine Mischstrategie. Seinen Auswertungen zufolge erlernen $40 \%$ der Auszubildenden Berufe, die primär nach dem Produktionsmotiv ausgebildet werden, $32 \%$ solche, die nach dem Investitionsmotiv ausgebildet werden und $28 \%$ solche, die nicht eindeutig zugeordnet werden können.

Büchel und Neubäumer (2001) wählen ein ähnliches Verfahren zur Untermauerung ihrer Hypothesen zur Segmentierung des Ausbildungsmarktes. Sie nutzen die Daten der BIBB-Kosten-Nutzen-Erhebung des Jahres 1991 (vgl. Bardeleben et al. 1995) aggregiert auf Branchenebene. Sie unterteilen den Ausbildungsmarkt in zwei Segmente, eines mit hohen Ausbildungskosten, hohem Übernahmeinteresse der Betriebe und ausgeprägten internen Arbeitsmärkten mit guten Entwicklungsmöglichkeiten für die Ausbildungsabsolventen. In dem anderen Segment gibt es nur geringe oder keine Nettoausbildungskosten und es wird über Bedarf ausgebildet. Die Absolventen des zweiten Sektors wechseln häufig nach der Ausbildung in Betriebe des ersten Sektors, um dort auf An- oder Ungelerntenstellen tätig zu sein. Büchel und Neubäumer (2001) ordnen die verschiedenen Branchen den beiden Sektoren zu und prüfen, ob die Kategorisierung überhaupt zutreffend ist und welchen Einfluss sie auf den Beschäftigungserfolg der Auszubildenden hat.

Auf Basis der Übernahmeentscheidung analysieren Beicht et al. (2004) und Mohrenweiser und Backes-Gellner (2010) das Verhältnis von Investitions- und Produktionsmotiv. Erstere kommen zu dem Ergebnis, dass etwa ein Drittel der Auszubildenden nach dem Produktionsmotiv ausgebildet werden. Letztere haben mit Daten des IABBetriebspanels berechnet, dass etwa $44 \%$ der ausbildenden Betriebe in Deutschland eine Investitionsstrategie verfolgen, während rund $19 \%$ nach dem Produktionsmotiv ausbilden. Die verbleibenden Betriebe können keinem Ausbildungsmotiv eindeutig zugeordnet werden.

Dionisius et al. (2009a) vergleichen die Bedeutung von Investitions- und Produktionsmotiv in Deutschland und der Schweiz. Die Länder eignen sich aufgrund der vergleichbaren Ausbildungssysteme besonders für einen Vergleich. Im Jahr 2000 wurden in beiden Länder eine Kosten-NutzenErhebung durchgeführt (vgl. Beicht et al. 2004 und Schweri et al. 2003). Dionisius et al. (2009a) finden eine deutli- che Evidenz dafür, dass das Investitionsmotiv in Deutschland eine weitere Verbreitung als in der Schweiz hat. Für ihre Untersuchung verwenden sie Einschätzungen und Bewertungen der Betriebe hinsichtlich der Ausbildungsmotive.

Mohrenweiser und Zwick (2009) verwenden den linkedemployer-emloyee Datensatz des Instituts für Arbeitsmarktund Berufsforschung (LIAB), um zu zeigen, dass eine Erhöhung des Anteils an Auszubildenden im Vergleich zu An- oder Ungelernten bei den Produktionsberufen des verarbeitenden Gewerbes zu niedrigeren Gewinnen des Betriebs führt, die Ausbildung also Kosten verursacht. Bei den kaufmännischen und den handwerklichen Berufen stellen sie steigende Gewinne, also einen Nutzen der Ausbildung, fest. Mit diesem indirekten Verfahren können sie über die Ausbildungsquote und die wirtschaftliche Entwicklung des Betriebs zeigen, dass für einen Teil der Betriebe keine zusätzlichen Kosten durch die Ausbildung entstehen und sie somit einem Produktionsmotiv folgen könnten.

Pfeifer et al. (2010) untersuchen, ob es in Deutschland eine Entwicklung hin zu einer stärker produktionsmotivierten Ausbildung gibt. Sie verwenden dazu die Daten der BIBB-Kosten-Nutzen-Erhebungen der Jahre 2000 und 2007 und kommen zu dem Ergebnis, dass es zu einem deutlichen Rückgang der Nettokosten gekommen ist, ursächlich hierfür ist vor allem ein Anstieg der Erträge. Gleichzeitig hat sich das Übernahmeinteresse der Betriebe aber nur leicht verringert. Sie können die These, dass das Produktionsmotiv eine stärkere Bedeutung erlangt hat, also nicht erhärten.

Der Organisation der Ausbildung am Arbeitsplatz und insbesondere der Aufteilung der Zeit, die die Auszubildenden im Betrieb verbringen, wurde in der ökonomischen Literatur bislang nur wenig Aufmerksamkeit geschenkt. In den Veröffentlichungen zu den BIBB-Kosten-NutzenErhebungen (vgl. Beicht et al. 2004 und Schönfeld et al. 2010) werden die Zeiten der Auszubildenden deskriptiv dargestellt, jedoch keine Analysen durchgeführt, welche Faktoren sie beeinflussen. Dabei wird zwischen Zeiten, in denen einfache produktive Tätigkeiten ausgeführt werden, die normalerweise An- oder Ungelernte durchführen, Zeiten, in denen Fachkräftetätigkeiten übernommen werden, und unproduktiven Zeiten, in denen keine für den Betrieb verwertbaren Produkte erstellt werden, unterschieden. Während der unproduktiven Zeiten sind die Auszubildenden in verschiedenen Kursen in einer Lehrwerkstatt, im innerbetrieblichen Unterricht oder auch bei externen Angeboten oder sie lernen selbstständig. Ausbildungsinhalte werden dabei nicht nur während der unproduktiven Zeiten vermittelt. Insbesondere durch das Durchführen von Fachkräftetätigkeiten findet ein Lernen im Arbeitsprozess statt. ${ }^{9}$

\footnotetext{
${ }^{9}$ Zur Diskussion in der Berufspädagogik siehe z.B. Heinemann und Rauner (2008) oder Reglin und Schöpf (2005).
} 
Dionisius et al. (2009b) identifizieren die Zeitaufteilung am Arbeitsplatz als einen wesentlichen Faktor zur Erklärung der Kostenunterschiede zwischen Deutschland und der Schweiz. Ihre Analysen basieren auf dem BIBB-CBS für das Jahr 2000 (vgl. Beicht et al. 2004) und einer fast identischen Erhebung der Universität Bern im gleichen Jahr (vgl. Schweri et al. 2003). In der Schweiz werden die Auszubildenden zu wesentlich größeren Anteilen im Produktionsprozess ausgebildet als in Deutschland, u.a. aus diesem Grund sind die Erträge höher und die Nettokosten entsprechend niedriger.

Fougère und Schwerdt (2002) untersuchen die Ausbildungsbeteiligung und den Einfluss der Anzahl an Auszubildenden auf den produktiven Output von Betrieben im Vergleich zwischen Deutschland und Frankreich unter Berücksichtigung der Betriebsgröße. Sie kommen zu dem Ergebnis, dass in kleinen und großen Betrieben die Ausbildung in erster Linie der Fachkräfterekrutierung dient, während in mittleren Betrieben die Auszubildenden stärker an der Produktion beteiligt werden. Auch die Differenz zwischen dem Lohn der An- oder Ungelernten und dem der Auszubildenden hat einen positiven Einfluss auf die Beteiligung an der Produktion. Eine direkte Messung der produktiven Zeiten der Auszubildenden findet aber nicht statt.

Zusammenfassend kann gesagt werden, dass die Betriebe nur wenige Faktoren der Ausbildungskosten direkt beeinflussen können. ${ }^{10}$ Es ist daher zu erwarten, dass die Betriebe, die dem Produktionsmotiv folgen, ihre Auszubildenden stärker in die produktiven Prozesse integrieren, um die entstehenden Kosten der Ausbildung mit diesen Erträgen abdecken zu können. Bei den investitionsorientierten Betrieben kann durch die Übernahme der Auszubildenden weiterer Nutzen generiert werden, folglich sind sie in geringerem Umfang auf die Erträge während der Ausbildungszeit angewiesen. Die Zeitaufteilung könnte in diesen Betrieben eher durch pädagogische Überlegungen geprägt sein, da eine hohe Ausbildungsqualität erzielt werden soll. Hypothese 1 lautet daher wie folgt:

Die produktiven Zeitanteile der Auszubildenden, also die Summe der Zeitanteile mit einfachen und Fachkräftetätigkeiten, fallen in produktionsorientierten Betrieben höher aus als in investitionsorientierten Betrieben.

Neben der strategischen Ausrichtung der Ausbildung innerhalb des Betriebs haben auch die Auszubildenden selbst bzw. ihre persönlichen Eigenschaften einen Effekt auf die Gestaltung der Ausbildung im Betrieb. Insbesondere die

\footnotetext{
${ }^{10}$ So sind insbesondere die Vergütungen der Auszubildenden, die etwa $60 \%$ der Bruttoausbildungskosten ausmachen, aufgrund tarifvertraglicher und gesetzlicher Vorgaben nur bedingt durch den Betrieb beeinflussbar.
}

Kompetenzen, die sie bereits mit in die Ausbildung bringen, sollten hierfür verantwortlich sein. Aus zwei Gründen ist ein stärkerer produktiver Einsatz besser qualifizierter Auszubildender für Fachkräftetätigkeiten möglich. Zum einen können sie bereits eine größere Zahl von Aufgaben übernehmen und diese auch in höherer Qualität ausführen. Zum anderen müssen sie in geringerem Umfang auf die Abschlussprüfungen vorbereitet werden. Bei den investierenden Betrieben kann erwartet werden, dass der höhere Anteil an Fachkräftetätigkeiten durch weniger unproduktive Zeiten ausgeglichen wird, da für besser Qualifizierte Ausbildungsmaßnahmen, die abseits des Produktionsprozesses stattfinden, reduziert werden könnten. Bei produktionsorientierten Betrieben ist eine Substitution der einfachen Tätigkeiten zu erwarten, wenn der Einsatz für Fachkräftetätigkeiten eine höhere Rendite verspricht. Bei den unproduktiven Zeiten kann vermutet werden, dass diese sich unabhängig von den allgemeinen schulischen Kompetenzen auf einem minimalen Niveau befinden. Hypothese 2 lautet daher wie folgt:

Auszubildende mit geringeren allgemeinen schulischen Kompetenzen werden in geringerem Umfang für Fachkräftetätigkeiten eingesetzt.

Aufgrund sich überlappender Effekte kann keine Einflussrichtung wirtschaftlicher Rahmenbedingungen vorhergesagt werden. Bei guten wirtschaftlichen Rahmenbedingungen sollten die produktiven Zeitanteile der Auszubildenden größer ausfallen, da auch ausreichend Arbeitsaufträge vorhanden sein sollten. Gleichzeitig kann es aber auch sein, dass bei Kapazitätsengpässen, die bei guter Auftragslage entstehen können, Arbeiten nicht von Auszubildenden durchgeführt werden können, da sie bei diesen Arbeiten betreut werden müssen und mehr Zeit benötigen als die betreuende Fachkraft. Daher kann es sinnvoll sein, dass die Fachkraft die Arbeiten selbst ausführt, während der Auszubildende nur zuschaut und die produktiven Zeitanteile der Auszubildenden daher geringer ausfallen. Die wirtschaftlichen Rahmenbedingungen dienen in diesem Artikel hingegen als Approximation externer Schocks. Für unerwartete Veränderungen, die einen Einfluss auf die zeitliche Organisation der Ausbildung haben können, kann so auf einzelbetrieblicher Ebene kontrolliert werden.

Im Verlauf der Ausbildung kann erwartet werden, dass die Auszubildenden stärker in produktive Prozesse integriert werden, insbesondere gilt dies für Tätigkeiten auf Fachkräfteniveau. Ob der Zusammenhang mit den Ausbildungsmotiven in allen Jahren gleich stark ist, kann nicht theoretisch hergeleitet werden. Der Einfluss der Berufe sollte aber zurückgehen, da vor allem zu Beginn der Ausbildung berufsspezifische Rahmenbedingungen, wie die Ausbildungsordnungen, die Einsetzbarkeit der Auszubildenden beeinflussen können. Im letzten Teil der Ausbildung sollten die Betriebe einen größeren Gestaltungsfreiraum haben. 
Tab. 1 Zeitaufteilung in Tagen pro Auszubildenden und Jahr

\begin{tabular}{lccc}
\hline & 1. Ausbildungsjahr & 2. Ausbildungsjahr & 3. Ausbildungsjahr \\
\hline Einfache Tätigkeiten & 63 & 51 & 43 \\
Fachkräftetätigkeiten & 33 & 57 & 73 \\
Unproduktive Tätigkeiten & 54 & 47 & 43 \\
Tage, die die Auszubildenden & 150 & 156 & 158 \\
dem Betrieb zur Verfügung & & & \\
stehen & 65 & 59 & 56 \\
Berufsschultage & 8 & 8 & 9 \\
Krankheitstage & 27 & 28 & 28 \\
Urlaubstage & 250 & 250 & 250 \\
Gesamt & 1145 & 1355 & 1245 \\
$N$ & & & \\
\hline
\end{tabular}

\section{Datenbasis}

Die Datenbasis für diesen Artikel ist die Kosten-NutzenErhebung des Bundesinstituts für Berufsbildung für das Jahr 2007 (BIBB-CBS 2007). Detaillierte Informationen zur Durchführung und Konzeption sowie Ergebnissen der Erhebung finden sich bei Schönfeld et al. (2010). An dieser repräsentativen Erhebung haben knapp 3000 Ausbildungsbetriebe teilgenommen, die einen der 51 am häufigsten ausgebildeten Berufe anbieten. In den Betrieben wurden die Personal- oder Ausbildungsverantwortlichen in persönlichen Interviews zu den Kosten und dem Nutzen der betrieblichen Ausbildung in einem vorher ausgewählten Ausbildungsberuf befragt. Neben den Fragen zu Kosten und Nutzen bietet der Datensatz eine Vielzahl von Informationen zu der Betriebsstruktur, den wirtschaftlichen Rahmenbedingungen, den Rekrutierungsstrategien und Einschätzungen der Interviewten zu den Themen Fachkräfterekrutierung und Ausbildung. Die Antworten der Betriebe beziehen sich dabei immer auf den Durchschnitt der relevanten Gruppe, also z.B. die Gruppe der Auszubildenden oder verschiedene Mitarbeitergruppen. Der Datensatz ist der einzige, der repräsentative Daten zum Ausbildungsverhalten der Ausbildungsbetriebe und zu Kosten und Nutzen der Ausbildung in Deutschland liefert.

Für die folgenden Analysen wurden die Betriebe des öffentlichen Dienstes ausgeschlossen, da sich in diesen Betrieben die Ausbildungsentscheidung und -organisation weniger stark als in Betrieben des privaten Sektors an ökonomischen Überlegungen ausrichtet. Daher ergibt sich eine Gesamtzahl von 2223 Ausbildungsbetrieben. Bei den deskriptiven Darstellungen werden Gewichtungsfaktoren verwendet, die auf die Gesamtheit der Ausbildungsbetriebe in Deutschland, ausgerichtet sind (für eine Erläuterung der Berechnung der Gewichte siehe Schönfeld et al. 2010). Die Randverteilungen zur Bestimmung der Gewichte stammen aus der Betriebsdatei der Bundesagentur für Arbeit.
Eine Besonderheit des Datensatzes ist es, dass viele der erhobenen Variablen für jedes Ausbildungsjahr einzeln erfragt wurden. Dies hat den Vorteil, dass Unterschiede, z.B. in der Ausbildungsorganisation, nach Ausbildungsjahren identifizierbar sind. Nachteilig ist allerdings, dass die Betriebe nur zu den Ausbildungsjahren befragt wurden, in denen sie auch Auszubildende beschäftigten. Die Angaben für die „fehlenden“ Jahre müssen daher geschätzt werden, bzw. es kann nur eine geringere Fallzahl pro Jahr betrachtet werden. ${ }^{11}$ Für diesen Artikel wurden die Angaben für die fehlenden Jahre auf Basis der vorhandenen Jahre mit multivariaten Verfahren geschätzt. Für die meisten Analysen wurden dann die Durchschnittswerte aller Ausbildungsjahre verwendet.

\section{Empirische Ergebnisse}

\subsection{Variablenkonstruktion und -deskription}

Im dualen System findet die Ausbildung bekanntlich zumindest an den Lernorten Betrieb und Berufsschule statt. In einigen Betrieben werden die Jugendlichen auch in Lehrwerkstätten (knapp $5 \%$ der Betriebe), durch innerbetrieblichen Unterricht (26\%) oder in externen Ausbildungsphasen (etwa $34 \%$ ) ausgebildet. Tabelle 1 zeigt, wie sich die Zeiten der Auszubildenden auf die einzelnen Lernorte, -formen und Abwesenheitsarten in den einzelnen Ausbildungsjahren $^{12}$ verteilen. Es werden pro Jahr nur die Betriebe einbezogen, die Auszubildende in diesem Ausbildungsjahr hatten.

\footnotetext{
${ }^{11}$ So haben z.B. nur 1145 der 2223 Betriebe mindestens einen Auszubildenden im ersten Ausbildungsjahr.

${ }^{12}$ Auf die Darstellung des vierten Ausbildungsjahres wird verzichtet, da es sich zum einen nur um ein halbes Jahr handelt und es zum anderen nur bei 12 Berufen ein viertes Jahr gibt. Die Ergebnisse sind daher nur schlecht mit den anderen Jahren vergleichbar.
} 
Unterschieden wird zwischen den vom Betrieb nicht beeinflussbaren Abwesenheitszeiten Berufsschule, Krankheit und Urlaub, und den Tagen, an denen der Betrieb über die Zeit der Auszubildenden verfügen kann. Bei letzteren kann wiederum zwischen drei Arten der Teilhabe der Auszubildenden am betrieblichen Wertschöpfungsprozess differenziert werden. Die Auszubildenden können entweder einfache Tätigkeiten ausführen, die ansonsten von An- oder Ungelernten durchgeführt werden, sie können Fachkräftetätigkeiten ausführen oder sie können ,unproduktiv“ sein. Unproduktiv meint dabei, dass keine für den Betrieb verwertbaren Leistungen oder Güter entstehen. Die Auszubildenden nehmen in dieser Zeit an verschiedenen Kursen oder Unterweisungen teil oder lernen im Selbststudium oder Übungsphasen.

Die Zahl der Urlaubs- und Krankheitstage unterscheidet sich nur geringfügig über die Ausbildungsjahre (vgl. Tab. 1). Die Auszubildenden sind durchschnittlich 8 bis 9 Tage krank und haben 27 bis 28 Tage Urlaub oder aus sonstigen Gründen frei. Bei den Berufsschultagen gibt es einen Rückgang vom ersten zum dritten Jahr, da in einigen Berufen zu Beginn der Ausbildung verstärkt in der Berufsschule ausgebildet wird. Im ersten Jahr sind es durchschnittlich 65 Berufsschultage, im dritten 56. Dem Betrieb stehen die Auszubildenden daher zwischen 150 und 158 Tage im Jahr zur Verfügung. Die Summe der unproduktiven Tage ist im ersten Jahr mit 54 Tagen am höchsten, im zweiten Jahr sind es 47, im dritten 43. Bei den einfachen Tätigkeiten ist der Verlauf ähnlich, von anfänglich 63 Tagen sinkt der Wert im dritten Jahr auf 43 Tage. Die Fachkräftetätigkeiten müssen demzufolge steigen. Sie erhöhen sich von 33 im ersten Jahr über 57 im zweiten auf 73 Tage im dritten Jahr. Insgesamt verbringen die Auszubildenden im dritten Jahr fast drei Viertel der Zeit, die sie dem Betrieb zur Verfügung stehen, mit produktiven Tätigkeiten, im ersten Jahr sind es $64 \%$. Für die weiteren Analysen wird nur noch die Aufteilung der für den Betrieb verfügbaren Zeit betrachtet.

Tabelle 2 zeigt diese Aufteilung nach verschiedenen betrieblichen Merkmalen. Im Durchschnitt über alle Betriebe und Ausbildungsjahre sind die Auszubildenden $30 \%$ der Zeit (im Sinne des Betriebsoutputs) unproduktiv. $36 \%$ der Zeit sind sie mit Fachkräftetätigkeiten und $34 \%$ mit einfachen Tätigkeiten beschäftigt. Bezüglich der Betriebsgröße zeigt sich, dass in kleineren Betrieben sowohl mehr Fachkräftetätigkeiten als auch mehr einfache Tätigkeiten ausgeübt werden. Der Wirtschaftszweig „Verwaltung/ Erziehung/Gesundheit" hat den höchsten Anteil an Fachkräftetätigkeiten $(40 \%)$, das verarbeitende Gewerbe den niedrigsten (34\%). Im Westen sind die Auszubildenden stärker mit einfachen Tätigkeiten beschäftigt und dafür seltener unproduktiv. Beim Betriebstyp gibt es nur geringe Unterschiede. $^{13}$

\footnotetext{
${ }^{13}$ Tabelle 8 im Anhang zeigt die Zeitaufteilung nach Ausbildungsberufen.
}

Die Motive werden nach den Antworten auf die folgende Frage des BIBB-CBS 2007 zugeordnet: „Welche Strategie verfolgt Ihr Betrieb im Hinblick auf eine mögliche Übernahme der Auszubildenden im Ausbildungsberuf?" Als Antwortmöglichkeiten waren ,auf jeden Fall weiterbeschäftigen“, „teilweise weiterbeschäftigen“ und „,nur in Ausnahmefällen weiterbeschäftigen" gegeben. Die erste Antwortmöglichkeit lässt dabei auf ein Investitionsmotiv des Betriebs schließen. Es lässt sich aber nicht ausschließen, dass es auch Betriebe gibt, die, obwohl sie alle Auszubildenden übernehmen möchten, auch darauf achten, dass die Bruttokosten der Ausbildung bereits durch die produktiven Leistungen der Auszubildenden gedeckt werden. Diesen Betrieben könnte man eine Doppelstrategie unterstellen, anhand der gewählten Motivvariablen lassen sie sich aber nicht identifizieren. Eindeutiger ist die Zuordnung bei den Betrieben, die nur im Ausnahmefall weiterbeschäftigen möchten. Sie folgen dem Produktionsmotiv, da sie nur so die Kosten der Ausbildung decken können. Bei der zweiten Antwortmöglichkeit kann keine eindeutige Zuordnung erfolgen, da der Anteil der Übernommenen offen ist. Tendenziell spricht die Aussage aber eher für ein Investitionsmotiv, da es z.B. die Strategie des Betriebs sein kann nur die besten Auszubildenden zu übernehmen und dieses Auswahlverfahren die Investition rechtfertigt. Im weiteren Vorgehen werden die drei Gruppen dennoch getrennt betrachtet. Die Betriebe, die teilweise weiterbeschäftigen, werden im Folgenden unter der Bezeichnung „Unbestimmtes Motiv“ geführt.

Insgesamt hat das Investitionsmotiv mit knapp $48 \%$ die größte Bedeutung, $23 \%$ der Betriebe werden dem Produktionsmotiv zugeordnet und bei etwa $29 \%$ ist das Motiv unklar (vgl. Tab. 3). Kleiner Betrieb folgen häufiger dem Produktionsmotiv, während bei den Großbetrieben $78 \%$ alle Auszubildende übernehmen möchten. Zwischen den Regionen gib es nur geringe Unterschiede. Auch bei den Wirtschaftszweigen sind sie eher gering. Im Bereich „Finanzdienstleistungen und Immobilien“ gibt es die größte Zustimmung zum Investitionsmotiv, der Bereich „Verwaltung/Erziehung/Gesundheit" hat den größten Anteil an Betrieben, die nur im Ausnahmefall übernehmen möchten.

In Tab. 4 wird der unmittelbare Zusammenhang zwischen den Ausbildungsmotiven und den Zeitanteilen dargestellt. Es werden jeweils OLS-Modelle auf die Zeitanteile mit den Motivdummies als einzige erklärende Variablen geschätzt. Es zeigt sich, dass in Betrieben des Investitionsmotivs (Referenzgruppe) die Auszubildenden weniger produktiv eingesetzt werden als bei den Betrieben mit unbestimmten oder Produktionsmotiv. Insbesondere bei den einfachen Tätigkeiten sind die Koeffizienten stark signifikant und positiv.

Die weiteren OLS-Schätzungen enthalten neben den Motivdummies die allgemeinen schulischen Kompetenzen der Auszubildenden. Zusätzlich wird der Anteil an Abiturienten aufgenommen, um dafür zu kontrollieren, dass die 
Tab. 2 Zeitaufteilung in Prozent nach Betriebsmerkmalen und Motiven

\begin{tabular}{|c|c|c|c|c|c|c|c|}
\hline & \multicolumn{2}{|c|}{ Anteil Unproduktive Zeiten } & \multicolumn{2}{|c|}{ Anteil Fachkräftetätigkeiten } & \multicolumn{2}{|c|}{ Anteil einfache Tätigkeiten } & \multirow[t]{2}{*}{$N$} \\
\hline & Mittelwert & Standardabw. & Mittelwert & Standardabw. & Mittelwert & Standardabw. & \\
\hline \multicolumn{8}{|l|}{ Ausbildungsmotiv } \\
\hline Investitionsmotiv & 32.31 & 19.11 & 34.74 & 16.96 & 32.95 & 16.08 & 1217 \\
\hline Unbestimmtes Motiv & 29.72 & 17.49 & 36.92 & 17.18 & 33.36 & 15.81 & 612 \\
\hline Produktionsmotiv & 27.22 & 16.45 & 37.21 & 17.91 & 35.58 & 16.18 & 394 \\
\hline \multicolumn{8}{|l|}{ Betriebsgrößenklassen } \\
\hline bis 9 Beschäftigte & 28.44 & 16.59 & 36.88 & 16.76 & 34.68 & 15.38 & 761 \\
\hline 10 bis 49 Besch. & 31.93 & 18.80 & 34.77 & 17.71 & 33.30 & 16.58 & 818 \\
\hline 50 bis 499 Besch. & 35.76 & 22.27 & 34.75 & 18.55 & 29.49 & 17.27 & 527 \\
\hline mehr als 499 Besch. & 48.01 & 23.16 & 29.44 & 15.91 & 22.55 & 15.40 & 117 \\
\hline \multicolumn{8}{|l|}{ Wirtschaftszweig ${ }^{\mathrm{a}}$} \\
\hline Verarbeitendes Gewerbe & 31.75 & 18.81 & 33.62 & 16.25 & 34.63 & 16.43 & 1007 \\
\hline Handel & 30.86 & 17.49 & 35.88 & 16.87 & 33.26 & 15.46 & 392 \\
\hline $\begin{array}{l}\text { Dienstleistungen } \\
\text { Gastgewerbe und } \\
\text { Infrastruktur }\end{array}$ & 28.19 & 17.80 & 38.16 & 18.42 & 33.65 & 15.67 & 475 \\
\hline $\begin{array}{l}\text { Finanzdienstleistungen } \\
\text { und Immobilien }\end{array}$ & 31.68 & 17.97 & 36.02 & 17.41 & 32.30 & 15.63 & 245 \\
\hline $\begin{array}{l}\text { Verwaltung/Erziehung/ } \\
\text { Gesundheit }\end{array}$ & 26.16 & 17.47 & 40.46 & 18.55 & 33.38 & 16.97 & 104 \\
\hline \multicolumn{8}{|l|}{ Region } \\
\hline Ost & 33.80 & 22.35 & 35.68 & 19.43 & 30.52 & 16.63 & 633 \\
\hline West & 29.74 & 17.20 & 35.99 & 16.85 & 34.27 & 15.88 & 1590 \\
\hline \multicolumn{8}{|l|}{ Betriebstyp } \\
\hline Einzelbetrieb & 30.04 & 17.80 & 36.04 & 17.24 & 33.92 & 15.82 & 1908 \\
\hline Zentrale & 33.93 & 21.41 & 32.68 & 15.37 & 33.39 & 17.72 & 156 \\
\hline Filiale/Zweigstelle & 32.98 & 20.53 & 36.61 & 18.78 & 30.41 & 17.87 & 159 \\
\hline Gesamt & 30.38 & 18.16 & 35.94 & 17.28 & 33.68 & 16.05 & 2223 \\
\hline
\end{tabular}

Quelle: BIBB-CBS 2007

${ }^{a}$ Ausgehend von der Klassifikation NACE Rev. 1.1 (2002) wurden die Wirtschaftszweige in fünf Kategorien unterteilt: Verarbeitendes Gewerbe, Handel, Verwaltung/Erziehung/Gesundheit, Dienstleistungen Gastgewerbe und Infrastruktur (insbesondere Gastgewerbe, Verkehr/Nachrichtenübermittlung und Energie-/Wasserversorgung) und Finanzdienstleistungen und Immobilien (insbesondere Kredit/Versicherungsgewerbe, Grundstücks-/Wohnungswesen und Vermietung beweglicher Sachen).

Bewertung der allgemeinen Kompetenzen möglicherweise durch den Schulabschluss beeinflusst wird. Betriebe könnten höhere Erwartungen bezüglich des Leistungsvermögens von Abiturienten haben und sie daher schlechter bewerten. Als Indikator für die allgemeinen schulischen Kompetenzen wurde ein Gesamtwert aus den Angaben der Betriebe über die schriftliche und mündliche Ausdrucksfähigkeit, die Beherrschung der Grundrechenarten, die Grundkenntnisse im IT-Bereich und die Problemlösefähigkeit der Auszubildenden gebildet. Bewertet werden konnte jeweils auf einer Skala von -2 ,sehr schlecht“ bis +2 ,sehr gut“. Da die Angaben zu den einzelnen Punkten hoch korreliert sind und die Betriebe nur zu den Punkten Angaben machen muss- ten, die für den Ausbildungsberuf relevant sind, wird der ungewichtete Durchschnitt dieser Angaben verwendet. GröBere Missinganteile gab es nur bei der Frage zu den ITGrundkenntnissen, die für einige Berufe nicht relevant sind. Weitere Kontrollvariablen sind Faktoren der wirtschaftlichen Rahmenbedingungen, die ebenfalls aus den Angaben des BIBB-CBS 2007 gebildet wurden. Hierfür wurden die Angaben der Betriebe zum aktuellen und zukünftigen Umsatz, ob auf Aufträge verzichtet wurde oder es erheblich längere Lieferzeiten gab und zur Kontrolle des Arbeitsmarktes, die Frage nach der Anzahl an und der Einsetzbarkeit von Fachkräften auf dem externen Arbeitsmarkt, verwendet. Der Umsatz und die Arbeitsmarkteinschätzungen konnten 
Tab. 3 Verteilung der Ausbildungsmotive nach Betriebsmerkmalen in Prozent

Quelle: BIBB-CBS 2007

Tab. 4 OLS-Regressionen der Zeitanteile

Robuste Standardfehler in zweiter Zeile; ${ }^{*} p<0.10$, ${ }^{* *} p<0.05,{ }^{* * *} p<0.01$ Quelle: BIBB-CBS 2007

\begin{tabular}{|c|c|c|c|}
\hline & Investitionsmotiv & Unbestimmtes Motiv & Produktionsmotiv \\
\hline \multicolumn{4}{|l|}{ Betriebsgrößenklasse } \\
\hline bis 9 Beschäftigte & 39.04 & 31.36 & 29.61 \\
\hline 10 bis 49 Beschäftigte & 55.88 & 27.53 & 16.59 \\
\hline 50 bis 499 Beschäftige & 69.31 & 25.14 & 5.55 \\
\hline $\begin{array}{l}\text { mehr als } 500 \\
\text { Beschäftigte }\end{array}$ & 77.84 & 15.72 & 6.44 \\
\hline \multicolumn{4}{|l|}{ Region } \\
\hline Ostdeutschland & 50.18 & 28.56 & 21.26 \\
\hline Westdeutschland & 47.14 & 29.59 & 23.27 \\
\hline \multicolumn{4}{|l|}{ Wirtschaftszweig } \\
\hline Gewerbe & 48.11 & 27.73 & 24.16 \\
\hline Handel & 48.82 & 30.89 & 20.29 \\
\hline $\begin{array}{l}\text { Dienstleistungen } \\
\text { Gastgewerbe und } \\
\text { Infrastruktur }\end{array}$ & 43.65 & 30.28 & 26.07 \\
\hline $\begin{array}{l}\text { Finanzdienstleistungen } \\
\text { und Immobilien }\end{array}$ & 54.49 & 26.52 & 19.00 \\
\hline $\begin{array}{l}\text { Verwaltung/Erziehung/ } \\
\text { Gesundheit }\end{array}$ & 39.66 & 34.20 & 26.14 \\
\hline Gesamt & 47.62 & 29.43 & 22.95 \\
\hline
\end{tabular}

\begin{tabular}{lccc}
\hline & $\begin{array}{l}\text { Anteil unproduktive } \\
\text { Zeiten }\end{array}$ & $\begin{array}{l}\text { Anteil } \\
\text { Fachkräftetätigkeiten }\end{array}$ & $\begin{array}{l}\text { Anteil einfache } \\
\text { Tätigkeiten }\end{array}$ \\
\hline Ausbildungsmotiv: Refg. Investitionsmotiv & & \\
Unbestimmtes Motiv & $-4.45^{* * *}$ & $1.48^{*}$ & $2.97^{* * *}$ \\
& 1.00 & 0.87 & 0.82 \\
Produktionsmotiv & $-8.69^{* * *}$ & $3.33^{* * *}$ & $5.36^{* * *}$ \\
& 1.09 & 1.05 & 0.97 \\
Konstante & $36.21^{* * *}$ & $33.68^{* * *}$ & $30.11^{* * *}$ \\
& 0.62 & 0.50 & 0.48 \\
$N$ & 2223 & 2223 & 2223 \\
$R^{2}$ & 0.026 & 0.005 & 0.016 \\
\hline
\end{tabular}

auf einer Skala von -2 bis +2 bewertet werden. Die Variable „Kapazitätsengpass“ nimmt den Wert 1 an, wenn es entweder einen Verzicht auf Aufträge oder erheblich längere Liefer- oder Wartezeiten für die Kunden gab. Die Betriebsstruktur wird durch Betriebsgrößenklassen (4 Klassen), den Ausbildungsberuf (47), den Wirtschaftszweig (5), die Region (West bzw. Ost) und den Betriebstyp (3) abgebildet. ${ }^{15}$

\subsection{Multivariate Ergebnisse}

In Tab. 5 sind die Ergebnisse der multivariaten Regressionen auf die Zeitanteile abgebildet. Um der vorliegenden leichten

${ }^{15}$ Tabelle 9 zeigt die deskriptiven Statistiken aller erklärenden Variablen.
Heteroskedastizität gerecht zu werden, werden robuste Standardfehler geschätzt. Da die Summe der drei Zeitanteile per Konstruktion $100 \%$ ergibt, ergeben sich die Koeffizienten einer Regression aus denen der beiden anderen. Die Unterscheidung der Zeitarten ist für die Interpretation der Ergebnisse von Bedeutung, daher werden alle drei Regressionen dargestellt. Im Vergleich zu den Ergebnissen aus Tabelle 4 sind die Koeffizienten der Motivdummies kleiner und auch nicht in allen Fällen signifikant. Unter Kontrolle der angegebenen Variablen ist der Koeffizient des Produktionsmotivs bei den unproduktiven Zeiten signifikant negativ. Hypothese 1 kann also gestützt werden. Es zeigt sich, dass in diesen Betrieben die Auszubildenden stärker mit Fachkräftetätigkeiten beschäftigt sind als in den Betrieben des Investitions- 
Tab. 5 OLS-Regressionen der Zeitanteile

\begin{tabular}{|c|c|c|c|}
\hline & Anteil unproduktive Zeiten & Anteil Fachkräftetätigkeiten & $\begin{array}{l}\text { Anteil einfache } \\
\text { Tätigkeiten }\end{array}$ \\
\hline \multicolumn{4}{|l|}{ Ausbildungsmotiv: Refg. Investitionsmotiv } \\
\hline \multirow[t]{2}{*}{ Unbestimmtes Motiv } & $-1.72 *$ & 0.89 & 0.83 \\
\hline & 0.95 & 0.88 & 0.82 \\
\hline \multirow[t]{2}{*}{ Produktionsmotiv } & $-3.37^{* * *}$ & $2.55^{* *}$ & 0.82 \\
\hline & 1.14 & 1.11 & 1.02 \\
\hline \multirow[t]{2}{*}{ Anteil Abiturienten } & 0.02 & -0.20 & 0.18 \\
\hline & 1.18 & 1.16 & 1.05 \\
\hline \multirow[t]{2}{*}{ Allgemeine schulische Kompetenzen } & $-1.88^{* * *}$ & $2.91^{* * *}$ & $-1.04^{* *}$ \\
\hline & 0.53 & 0.51 & 0.49 \\
\hline \multirow{2}{*}{$\begin{array}{l}\text { Einschätzung der wirtschaftlichen Situation } \\
\text { in Bezug auf Umsatz }\end{array}$} & 0.64 & 0.67 & $-1.30^{* * *}$ \\
\hline & 0.55 & 0.50 & 0.47 \\
\hline \multirow{2}{*}{$\begin{array}{l}\text { Veränderung der wirtschaftlichen Situation in } \\
\text { den nächsten } 3 \text { Jahren }\end{array}$} & 0.05 & 0.09 & -0.14 \\
\hline & 0.58 & 0.54 & 0.49 \\
\hline \multirow[t]{2}{*}{ Kapazitätsengpässe } & -0.07 & 0.19 & -0.12 \\
\hline & 0.87 & 0.80 & 0.73 \\
\hline \multirow{2}{*}{$\begin{array}{l}\text { Anzahl Arbeitskräfte auf dem externen } \\
\text { Arbeitsmarkt }\end{array}$} & -0.03 & -0.20 & 0.23 \\
\hline & 0.40 & 0.38 & 0.35 \\
\hline \multirow[t]{2}{*}{ Einsetzbarkeit der externen Fachkräfte } & 0.33 & -0.52 & 0.19 \\
\hline & 0.47 & 0.43 & 0.40 \\
\hline \multicolumn{4}{|l|}{ Betriebsgrößenklassen: Refg.: $<10$ Beschäftigte } \\
\hline \multirow[t]{2}{*}{ 10-49 Besch. } & 1.07 & -0.79 & -0.28 \\
\hline & 1.00 & 0.95 & 0.88 \\
\hline \multirow[t]{2}{*}{ 50-499 Besch. } & 2.25 & 0.27 & $-2.51^{* *}$ \\
\hline & 1.37 & 1.24 & 1.11 \\
\hline \multirow[t]{2}{*}{500 und mehr Besch. } & $11.10^{* * *}$ & -2.10 & $-9.00^{* * *}$ \\
\hline & 2.34 & 1.81 & 1.83 \\
\hline \multicolumn{4}{|l|}{ Beruf: Refg. Elektroniker/in für Betriebstechnik } \\
\hline \multirow[t]{2}{*}{ Landwirt/in } & $-14.43^{* * *}$ & 4.08 & $10.34^{* * *}$ \\
\hline & 4.25 & 3.09 & 3.37 \\
\hline \multirow[t]{2}{*}{ Gärtner/in } & $-19.10^{* * *}$ & $8.60^{* * *}$ & $10.49^{* * *}$ \\
\hline & 3.56 & 2.81 & 3.20 \\
\hline \multirow[t]{2}{*}{ Florist/in } & $-20.32^{* * *}$ & $6.64^{* *}$ & $13.68^{* * *}$ \\
\hline & 3.55 & 2.76 & 2.97 \\
\hline \multirow[t]{2}{*}{ Chemikant/in } & $7.52^{*}$ & -4.29 & -3.22 \\
\hline & 4.24 & 2.95 & 2.96 \\
\hline \multirow{2}{*}{$\begin{array}{l}\text { Mediengestalter/in für Digital- und } \\
\text { Printmedien }\end{array}$} & $-14.11^{* * *}$ & $13.69^{* * *}$ & 0.42 \\
\hline & 4.54 & 4.36 & 3.64 \\
\hline \multirow[t]{2}{*}{ Drucker/in } & $-18.78^{* * *}$ & $12.13^{* * *}$ & $6.65^{* *}$ \\
\hline & 3.77 & 3.21 & 3.28 \\
\hline \multirow[t]{2}{*}{ Metallbauer/in } & $-9.85^{* *}$ & $10.37^{* * *}$ & -0.52 \\
\hline & 3.97 & 3.06 & 2.55 \\
\hline \multirow{2}{*}{$\begin{array}{l}\text { Anlagenmechaniker/in für Sanitär-, } \\
\text { Heizungs- und Klimatechnik }\end{array}$} & $-9.24^{* *}$ & $6.31^{* *}$ & 2.93 \\
\hline & 3.82 & 2.78 & 2.77 \\
\hline \multirow[t]{2}{*}{ Industriemechaniker/in } & 3.10 & -0.11 & -2.99 \\
\hline & 3.83 & 2.47 & 2.55 \\
\hline
\end{tabular}


Tab. 5 (Fortsetzung)

\begin{tabular}{|c|c|c|c|}
\hline & Anteil unproduktive Zeiten & Anteil Fachkräftetätigkeiten & $\begin{array}{l}\text { Anteil einfache } \\
\text { Tätigkeiten }\end{array}$ \\
\hline \multirow[t]{2}{*}{ Kraftfahrzeugmechatroniker/in } & -2.47 & 2.70 & -0.23 \\
\hline & 4.26 & 3.33 & 2.85 \\
\hline \multirow[t]{2}{*}{ Werkzeugmechaniker/in } & 2.58 & 1.09 & -3.67 \\
\hline & 3.86 & 2.60 & 2.52 \\
\hline \multirow[t]{2}{*}{ Zahntechniker/in } & $-8.19^{*}$ & 5.43 & 2.77 \\
\hline & 4.63 & 3.63 & 3.60 \\
\hline \multirow[t]{2}{*}{ Augenoptiker/in } & -5.90 & 4.89 & 1.01 \\
\hline & 5.01 & 3.38 & 3.25 \\
\hline \multirow{2}{*}{$\begin{array}{l}\text { Elektroniker/in -Energie- und } \\
\text { Gebäudetechnik }\end{array}$} & $-10.56^{* * *}$ & $7.60^{* *}$ & 2.96 \\
\hline & 3.86 & 3.01 & 2.76 \\
\hline \multirow{2}{*}{$\begin{array}{l}\text { Informations- und } \\
\text { Telekommunikationssystem-Elektroniker/in }\end{array}$} & $-12.15^{* * *}$ & $5.93^{*}$ & $6.22^{* *}$ \\
\hline & 3.85 & 3.22 & 2.89 \\
\hline \multirow[t]{2}{*}{ Mechatroniker/in } & 2.41 & -0.72 & -1.70 \\
\hline & 4.06 & 2.66 & 2.76 \\
\hline \multirow[t]{2}{*}{ Informationselektroniker/in } & $-9.31^{* * *}$ & $6.04^{* *}$ & 3.27 \\
\hline & 3.61 & 3.04 & 2.67 \\
\hline \multirow[t]{2}{*}{ Bäcker/in } & $-23.03^{* * *}$ & $11.79^{* * *}$ & $11.24^{* * *}$ \\
\hline & 3.69 & 3.10 & 3.00 \\
\hline \multirow[t]{2}{*}{ Fleischer/in } & $-19.96^{* * *}$ & $14.03^{* * *}$ & $5.93^{*}$ \\
\hline & 4.21 & 3.36 & 3.24 \\
\hline \multirow[t]{2}{*}{ Koch/Köchin } & $-26.84^{* * *}$ & $11.31^{* * *}$ & $15.53^{* * *}$ \\
\hline & 3.45 & 3.21 & 2.95 \\
\hline \multirow[t]{2}{*}{ Maurer/in } & -7.22 & -2.32 & $9.53^{* *}$ \\
\hline & 5.24 & 3.55 & 3.75 \\
\hline \multirow[t]{2}{*}{ Tischler/in } & -6.29 & -0.25 & $6.53^{* *}$ \\
\hline & 3.84 & 3.21 & 3.31 \\
\hline \multirow[t]{2}{*}{ Maler/in und Lackierer/in } & $-17.19^{* * *}$ & $7.75^{* *}$ & $9.44^{* * *}$ \\
\hline & 3.67 & 3.07 & 3.02 \\
\hline \multirow[t]{2}{*}{ Chemielaborant/in } & -5.78 & $6.04^{*}$ & -0.26 \\
\hline & 4.18 & 3.32 & 3.17 \\
\hline \multirow{2}{*}{ Bauzeichner/in } & $-11.62^{* * *}$ & $5.76^{* *}$ & $5.86^{* *}$ \\
\hline & 3.61 & 2.81 & 2.63 \\
\hline \multirow[t]{2}{*}{ Kaufmann/-frau im Groß- und Außenhandel } & $-22.39^{* * *}$ & $9.53^{* * *}$ & $12.87^{* * *}$ \\
\hline & 3.53 & 3.04 & 2.89 \\
\hline \multirow[t]{2}{*}{ Kaufmann/-frau im Einzelhandel } & $-21.89^{* * *}$ & $10.57^{* * *}$ & $11.33^{* * *}$ \\
\hline & 3.74 & 3.61 & 3.35 \\
\hline \multirow{2}{*}{$\begin{array}{l}\text { Fachverkäufer/in im } \\
\text { Nahrungsmittelhandwerk }\end{array}$} & $-19.95^{* * *}$ & $15.61^{* * *}$ & 4.34 \\
\hline & 3.58 & 2.86 & 3.02 \\
\hline \multirow[t]{2}{*}{ Bankkaufmann/-frau } & $-16.06^{* * *}$ & $7.68^{*}$ & $8.38^{* *}$ \\
\hline & 4.31 & 3.98 & 3.47 \\
\hline \multirow[t]{2}{*}{ Versicherungskaufmann/-frau } & $-8.05^{*}$ & 5.17 & 2.87 \\
\hline & 4.36 & 3.42 & 3.32 \\
\hline \multirow[t]{2}{*}{ Reiseverkehrskaufmann/-frau } & $-17.08^{* * *}$ & $12.11^{* * *}$ & $4.97^{*}$ \\
\hline & 3.97 & 3.21 & 2.77 \\
\hline \multirow[t]{2}{*}{ Veranstaltungskaufmann/-frau } & $-24.93^{* * *}$ & $23.51^{* * *}$ & 1.41 \\
\hline & 4.78 & 4.91 & 4.47 \\
\hline
\end{tabular}


Tab. 5 (Fortsetzung)

\begin{tabular}{|c|c|c|c|}
\hline & Anteil unproduktive Zeiten & Anteil Fachkräftetätigkeiten & $\begin{array}{l}\text { Anteil einfache } \\
\text { Tätigkeiten }\end{array}$ \\
\hline \multirow[t]{2}{*}{ Fachkraft für Lagerlogistik } & $-22.30^{* * *}$ & $5.28^{*}$ & $17.02^{* * *}$ \\
\hline & 3.70 & 3.09 & 3.43 \\
\hline \multirow[t]{2}{*}{ Steuerfachangestellte/r } & $-20.55^{* * *}$ & $11.13^{* * *}$ & $9.42^{* * *}$ \\
\hline & 3.39 & 3.23 & 2.84 \\
\hline \multirow{2}{*}{$\begin{array}{l}\text { Informations- und } \\
\text { Telekommunikationssystem-Kaufmann/-frau }\end{array}$} & $-13.75^{* * *}$ & $6.19^{* *}$ & $7.56^{* * *}$ \\
\hline & 3.62 & 2.55 & 2.64 \\
\hline \multirow[t]{2}{*}{ Informatikkaufmann/-frau } & $-16.36^{* * *}$ & $12.23^{* * *}$ & 4.13 \\
\hline & 3.73 & 3.32 & 2.94 \\
\hline \multirow[t]{2}{*}{ Fachinformatiker/in -Systemintegration } & $-12.13^{* * *}$ & $7.94^{* * *}$ & 4.18 \\
\hline & 3.58 & 2.91 & 2.91 \\
\hline \multirow[t]{2}{*}{ Rechtsanwaltsfachangestellte/r } & $-23.35^{* * *}$ & $12.61^{* * *}$ & $10.74^{* * *}$ \\
\hline & 4.08 & 3.45 & 3.67 \\
\hline \multirow[t]{2}{*}{ Kaufmann/-frau für Bürokommunikation } & $-20.02^{* * *}$ & $8.05^{* *}$ & $11.96^{* * *}$ \\
\hline & 3.87 & 3.28 & 2.96 \\
\hline \multirow[t]{2}{*}{ Bürokaufmann/-frau (Industrie und Handel) } & $-19.12^{* * *}$ & $8.15^{* * *}$ & $10.97^{* * *}$ \\
\hline & 3.56 & 2.58 & 2.46 \\
\hline \multirow[t]{2}{*}{ Bürokaufmann/-frau (Handwerk) } & $-17.38^{* * *}$ & $8.37^{* * *}$ & $9.00^{* * *}$ \\
\hline & 3.47 & 2.83 & 3.03 \\
\hline \multirow[t]{2}{*}{ Industriekaufmann/-frau } & $-20.86^{* * *}$ & $11.16^{* * *}$ & $9.70^{* * *}$ \\
\hline & 3.26 & 2.70 & 2.49 \\
\hline \multirow[t]{2}{*}{ (Zahn-)Medizinische/r Fachangestellte/r ${ }^{\mathrm{a}}$} & $-25.08^{* * *}$ & $17.03^{* * *}$ & $8.05^{* *}$ \\
\hline & 4.60 & 3.95 & 3.86 \\
\hline \multirow[t]{2}{*}{ Friseur/in } & $-14.26^{* * *}$ & $7.02^{*}$ & $7.24^{* *}$ \\
\hline & 4.31 & 3.89 & 3.60 \\
\hline \multirow[t]{2}{*}{ Hotelfachmann/-frau } & $-24.17^{* * *}$ & $16.47^{* * *}$ & $7.70^{* * *}$ \\
\hline & 3.62 & 3.30 & 2.96 \\
\hline \multirow[t]{2}{*}{ ÖD-Berufe ${ }^{b}$} & -3.86 & -2.41 & $6.27^{* *}$ \\
\hline & 3.98 & 3.22 & 2.95 \\
\hline \multicolumn{4}{|l|}{ Wirtschaftszweig: Refg. Verarbeitendes Gewerbe } \\
\hline \multirow[t]{2}{*}{ Handel } & -0.33 & $2.55^{*}$ & -2.22 \\
\hline & 1.52 & 1.35 & 1.36 \\
\hline \multirow{2}{*}{$\begin{array}{l}\text { Dienstleistungen Gastgewerbe und } \\
\text { Infrastruktur }\end{array}$} & 1.68 & 0.96 & $-2.64^{* *}$ \\
\hline & 1.51 & 1.36 & 1.26 \\
\hline \multirow[t]{2}{*}{ Finanzdienstleistungen und Immobilien } & 2.68 & -0.13 & -2.55 \\
\hline & 1.80 & 1.74 & 1.56 \\
\hline \multirow[t]{2}{*}{ Verwaltung/Erziehung/Gesundheit } & 1.98 & 0.45 & -2.43 \\
\hline & 3.33 & 2.67 & 2.47 \\
\hline \multirow[t]{2}{*}{ Region (West = 1) } & $-3.98^{* * *}$ & 0.00 & $3.97^{* * *}$ \\
\hline & 0.98 & 0.87 & 0.79 \\
\hline \multicolumn{4}{|l|}{ Betriebstyp: Refg. Einzelbetrieb } \\
\hline \multirow[t]{2}{*}{ Zentrale } & $3.42^{*}$ & $-3.52^{* *}$ & 0.10 \\
\hline & 1.78 & 1.42 & 1.47 \\
\hline \multirow[t]{2}{*}{ Filiale/Zweigstelle } & 2.31 & -0.81 & -1.50 \\
\hline & 1.66 & 1.59 & 1.45 \\
\hline
\end{tabular}


Tab. 5 (Fortsetzung)

\begin{tabular}{lccc}
\hline & Anteil unproduktive Zeiten & Anteil Fachkräftetätigkeiten & $\begin{array}{c}\text { Anteil einfache } \\
\text { Tätigkeiten }\end{array}$ \\
\hline Konstante & $48.41^{* * *}$ & $25.37^{* * *}$ & $26.22^{* * *}$ \\
$N$ & 2.99 & 2.14 & 2.16 \\
$R^{2}$ & 2223 & 2223 & 2223 \\
\hline
\end{tabular}

Robuste Standardfehler in zweiter Zeile; ${ }^{*} p<0.10,{ }^{* *} p<0.05,{ }^{* * *} p<0.01$

Quelle: BIBB-CBS 2007

${ }^{a}$ Die zahnmedizinischen und medizinischen Fachangestellten wurden zusammengefasst

${ }^{b}$ Berufe des Ausbildungsbereichs Öffentlicher Dienst werden teilweise auch in der Privatwirtschaft ausgebildet. Die Berufe des öffentlichen Dienst (Vermessungstechniker/in, Sozialversicherungsfachangestellte/r, Verwaltungsfachangestellte/r und Fachangestellte/r für Bürokommunikation) wurden auf Grund der geringen Fallzahl zusammengefasst

motivs. Der Koeffizient in der Regression der einfachen Tätigkeiten ist positiv, aber insignifikant. Für die Betriebe mit unbestimmtem Motiv sind die Koeffizienten kleiner und nur bei den unproduktiven Zeiten schwach signifikant.

Bezüglich Hypothese 2 zeigt sich, dass bei besseren allgemeinen schulischen Kompetenzen der Auszubildenden diese verstärkt für Fachkräftetätigkeiten eingesetzt werden. Dafür ist sowohl der Koeffizient bei den unproduktiven Zeiten als auch der bei den einfachen Tätigkeiten signifikant negativ. Kausalität kann aber nicht getestet werden. Daher könnte es sein, dass der positive Zusammenhang darauf zurückzuführen ist, dass Betriebe, die einen stärkeren produktiven Einsatz anstreben, eine stärkere Selektion der Bewerber auf Ausbildungsplätze durchführen. Die Koeffizienten der Variable „Anteil Abiturienten“ sind nicht signifikant.

Von den Variablen, die die wirtschaftlichen Rahmenbedingungen abbilden, ist nur der aktuelle Umsatz bei den einfachen Tätigkeiten negativ signifikant. Bei schlechterem Umsatz werden die Auszubildenden stärker für einfache Tätigkeiten eingesetzt. Wirtschaftliche Rahmenbedingungen spielen insgesamt eine unbedeutende Rolle bei der Entscheidung, wie die Auszubildenden im Betrieb eingesetzt werden.

In Betrieben mit 500 und mehr Beschäftigten ist der Anteil der unproduktiven Zeiten wesentlich höher, der Anteil an einfachen Tätigkeiten niedriger. In Großbetrieben ist das Kennenlernen des Betriebs, das einem mehrfachen Wechsel des Arbeitsbereichs beinhaltet, häufig Teil der Ausbildung. Daher fallen für die Auszubildenden öfter Zeiten an, in denen sie zunächst mit den Aufgaben des jeweiligen Arbeitsbereichs vertraut gemacht werden müssen und sie somit nicht produktiv eingesetzt werden können. Auch Phasen in Lehrwerkstätten oder im innerbetrieblichen Unterricht sind in Großbetrieben nicht unüblich. In der Regression der Fachkräftetätigkeiten sind die Koeffizienten der Betriebsgrößenklassen alle insignifikant.
Den stärksten Einfluss auf die Zeitaufteilung hat aber der ausgebildete Beruf, da die Betriebe zum einen die Vorgaben der jeweiligen Ausbildungsordnung erfüllen müssen und zum anderen wirtschaftliche und mitunter auch gesundheitliche Risiken den produktiven Einsatz der Auszubildenden einschränken können. Es zeigt sich, dass der gewählte Referenzberuf „Elektroniker/-in für Betriebstechnik“ überdurchschnittlich stark unproduktiv ausgebildet wird. Nur der Koeffizient des Berufs „Chemikant/-in“ ist in der Regression der unproduktiven Zeiten positiv signifikant. Die Chemieberufe sind Beispiele für Berufe in denen es notwendig ist, dass die Auszubildenden in relativ großem Umfang unproduktiv ausgebildet werden, da sowohl gesundheitliche als auch unternehmerische Risiken bei Fehlverhalten bestehen. Die kaufmännischen Berufe hingegen haben größtenteils geringe Anteile an unproduktiven Zeiten, eine Ausnahme ist der Beruf „Versicherungskaufmann/-frau“. Den geringsten Anteil an unproduktiven Zeiten hat der Beruf „Koch/Köchin“. Bei den Berufen „Maurer/-in“ und „Tischler/-in“ ist der Anteil vergleichsweise hoch, da in diesen Berufen die Auszubildenden relativ häufig und auch zeitintensiv in überbetrieblichen Ausbildungseinrichtungen ausgebildet werden. Die Verteilung auf die produktiven Zeiten lässt sich weniger gut nach Berufen erklären. Dies könnte daran liegen, dass je nach Beruf die Differenzierung zwischen einfachen und Fachkräftetätigkeiten unterschiedlich ausfällt. Den höchsten Anteil an Fachkräftetätigkeiten hat der Beruf „Veranstaltungskaufmann/-frau“, den niedrigsten der Beruf „Chemikant/-in“. ${ }^{16}$ Den größten Koeffizienten bei den einfachen Tätigkeiten hat die „Fachkraft für Lagerlogistik“, den kleinsten, aber nicht signifikanten, der Beruf „Werkzeugmechaniker/in“.

Mit 24,4\% ist die erklärte Varianz bei den unproduktiven Zeiten am größten. Bei den produktiven Zeiten liegen die

\footnotetext{
${ }^{16}$ Der Beruf „Chemikant/in“ unterscheidet sich allerdings nicht signifikant vom Referenzberuf.
} 
Tab. 6 Nach Motiven getrennte OLS-Regressionen Anteile der unproduktiven Zeiten

\begin{tabular}{|c|c|c|c|}
\hline & Investitionsmotiv & Unbestimmtes Motiv & Produktionsmotiv \\
\hline \multirow[t]{2}{*}{ Anteil Abiturienten } & -0.09 & 0.68 & -3.64 \\
\hline & 1.61 & 2.33 & 3.07 \\
\hline \multirow[t]{2}{*}{ Allgemeine schulische Kompetenzen } & $-2.20^{* * *}$ & $-1.94^{* *}$ & -0.40 \\
\hline & 0.74 & 0.97 & 1.21 \\
\hline \multirow{2}{*}{$\begin{array}{l}\text { Einschätzung der wirtschaftlichen Situation } \\
\text { in Bezug auf Umsatz }\end{array}$} & $1.64^{* *}$ & -0.39 & -0.05 \\
\hline & 0.80 & 1.09 & 1.05 \\
\hline \multirow{2}{*}{$\begin{array}{l}\text { Veränderung der wirtschaftlichen Situation in } \\
\text { den nächsten } 3 \text { Jahren }\end{array}$} & -0.53 & 0.57 & 0.92 \\
\hline & 0.84 & 1.20 & 1.22 \\
\hline \multirow[t]{2}{*}{ Kapazitätsengpässe } & -0.30 & 1.33 & -1.33 \\
\hline & 1.26 & 1.59 & 2.01 \\
\hline \multirow{2}{*}{$\begin{array}{l}\text { Anzahl Arbeitskräfte auf dem externen } \\
\text { Arbeitsmarkt }\end{array}$} & -0.33 & 0.46 & -0.14 \\
\hline & 0.58 & 0.73 & 0.96 \\
\hline \multirow[t]{2}{*}{ Einsetzbarkeit der externen Fachkräfte } & 0.08 & 0.78 & -0.46 \\
\hline & 0.66 & 0.91 & 1.22 \\
\hline \multicolumn{4}{|l|}{ Betriebsgrößenklassen: Refg.: $<10$ Beschäftigte } \\
\hline \multirow[t]{2}{*}{ 10-49 Besch. } & 0.69 & -0.40 & 2.18 \\
\hline & 1.52 & 1.86 & 2.27 \\
\hline \multirow[t]{2}{*}{ 50-499 Besch. } & 2.63 & 0.85 & 1.42 \\
\hline & 1.87 & 2.41 & 5.45 \\
\hline \multirow[t]{2}{*}{500 und mehr Besch. } & $11.78^{* * *}$ & $10.97^{* *}$ & -18.75 \\
\hline & 2.90 & 4.88 & 11.49 \\
\hline Beruf & $\mathrm{Ja}$ & $\mathrm{Ja}$ & $\mathrm{Ja}$ \\
\hline Wirtschaftszweig & $\mathrm{Ja}$ & $\mathrm{Ja}$ & $\mathrm{Ja}$ \\
\hline Betriebstyp & $\mathrm{Ja}$ & $\mathrm{Ja}$ & $\mathrm{Ja}$ \\
\hline \multirow[t]{2}{*}{ Region (West = 1) } & $-3.27^{* *}$ & $-4.17^{* *}$ & $-6.43^{* * *}$ \\
\hline & 1.40 & 1.87 & 2.38 \\
\hline \multirow[t]{2}{*}{ Konstante } & $44.51^{* * *}$ & $58.90^{* * *}$ & $45.87^{* * *}$ \\
\hline & 3.56 & 7.39 & 15.34 \\
\hline$N$ & 1217 & 612 & 394 \\
\hline$R^{2}$ & 0.243 & 0.305 & 0.312 \\
\hline
\end{tabular}

Robuste Standardfehler in zweiter Zeile; ${ }^{*} p<0.10,{ }^{* *} p<0.05,{ }^{* * *} p<0.01$

Quelle: BIBB-CBS 2007

Werte des $R^{2}$ mit 12,2 bzw. $15,1 \%$ deutlich niedriger. Dies könnte daran liegen, dass für die Betriebe die Unterscheidung zwischen Fachkräftetätigkeiten und einfachen Tätigkeiten nicht immer eindeutig zu treffen ist.

Um zu untersuchen, ob die festgestellten Korrelationen bei den allgemeinen schulischen Kompetenzen sich bezüglich der Motive unterscheiden, wird das Modell der unproduktiven Zeiten getrennt nach den Motiven geschätzt (vgl. Tab. 6). Der Koeffizient der allgemeinen schulischen Kompetenzen ist in allen drei Fällen negativ, beim Produktionsmotiv aber betragsmäßig kleiner und nicht signifikant. In den produktionsorientierten Betrieben haben die allgemei- nen Kompetenzen also eine geringere Bedeutung bei der Frage in welchem Umfang die Auszubildenden in unproduktiven Phasen ausgebildet werden. Ein möglicher Grund hierfür könnte sein, dass die produktionsorientierten Betriebe unabhängig von den Kompetenzen ihrer Auszubildenden die Möglichkeiten zum produktiven Einsatz maximal ausnutzen. Die Kompetenzen haben in diesen Betrieben nur einen Einfluss auf die Verteilung zwischen einfachen und Fachkräftetätigkeiten.

In einem letzten Schritt werden die Modelle getrennt nach Ausbildungsjahren geschätzt. Es werden nur die Regressionen der unproduktiven Zeiten dargestellt (vgl. Tab. 7). 
Tab. 7 Nach Ausbildungsjahren getrennte OLS-Regressionen der Anteile der unproduktiven Zeiten

\begin{tabular}{|c|c|c|c|}
\hline & 1. Ausbildungsjahr & 2. Ausbildungsjahr & 3. Ausbildungsjahr \\
\hline \multicolumn{4}{|l|}{ Ausbildungsmotiv: Refg. Investitionsmotiv } \\
\hline \multirow[t]{2}{*}{ Unbestimmtes Motiv } & $-2.41^{* *}$ & $-1.93^{*}$ & -1.41 \\
\hline & 1.07 & 1.02 & 0.97 \\
\hline \multirow[t]{2}{*}{ Produktionsmotiv } & $-3.58^{* * *}$ & $-3.45^{* * *}$ & $-3.16^{* * *}$ \\
\hline & 1.23 & 1.21 & 1.15 \\
\hline \multirow[t]{2}{*}{ Anteil Abiturienten } & -0.13 & 0.30 & -0.19 \\
\hline & 1.29 & 1.24 & 1.16 \\
\hline \multirow[t]{2}{*}{ Allgemeine schulische Kompetenzen } & $-1.63^{* * *}$ & $-1.85^{* * *}$ & $-2.15^{* * *}$ \\
\hline & 0.57 & 0.56 & 0.55 \\
\hline \multirow{2}{*}{$\begin{array}{l}\text { Einschätzung der wirtschaftlichen Situation } \\
\text { in Bezug auf Umsatz }\end{array}$} & $1.30^{* *}$ & 0.45 & 0.25 \\
\hline & 0.62 & 0.58 & 0.55 \\
\hline \multirow{2}{*}{$\begin{array}{l}\text { Veränderung der wirtschaftlichen Situation in } \\
\text { den nächsten } 3 \text { Jahren }\end{array}$} & -0.18 & 0.20 & 0.34 \\
\hline & 0.65 & 0.62 & 0.59 \\
\hline \multirow[t]{2}{*}{ Kapazitätsengpässe } & 0.53 & -0.54 & -0.26 \\
\hline & 0.98 & 0.92 & 0.89 \\
\hline \multirow{2}{*}{$\begin{array}{l}\text { Anzahl Arbeitskräfte auf dem externen } \\
\text { Arbeitsmarkt }\end{array}$} & 0.06 & -0.08 & -0.07 \\
\hline & 0.45 & 0.42 & 0.40 \\
\hline \multirow[t]{2}{*}{ Einsetzbarkeit der externen Fachkräfte } & 0.16 & 0.07 & 0.76 \\
\hline & 0.52 & 0.49 & 0.48 \\
\hline \multicolumn{4}{|l|}{ Betriebsgrößenklassen: Refg.: $<10$ Beschäftigte } \\
\hline \multirow[t]{2}{*}{ 10-49 Besch. } & 1.77 & 0.92 & $1.66^{*}$ \\
\hline & 1.09 & 1.07 & 1.00 \\
\hline \multirow[t]{2}{*}{ 50-499 Besch. } & $3.25^{* *}$ & 1.83 & 1.85 \\
\hline & 1.54 & 1.46 & 1.37 \\
\hline \multirow[t]{2}{*}{500 und mehr Besch. } & $15.32^{* * *}$ & $11.58^{* * *}$ & $7.51^{* * *}$ \\
\hline & 2.75 & 2.49 & 2.56 \\
\hline Beruf & $\mathrm{Ja}$ & $\mathrm{Ja}$ & $\mathrm{Ja}$ \\
\hline Wirtschaftszweig & $\mathrm{Ja}$ & $\mathrm{Ja}$ & $\mathrm{Ja}$ \\
\hline Betriebstyp & $\mathrm{Ja}$ & $\mathrm{Ja}$ & $\mathrm{Ja}$ \\
\hline \multirow[t]{2}{*}{ Region $($ West $=1)$} & $-4.50^{* * *}$ & $-4.90^{* * *}$ & $-3.89^{* * *}$ \\
\hline & 1.05 & 1.04 & 1.00 \\
\hline \multirow[t]{2}{*}{ Konstante } & $59.58^{* * *}$ & $49.74^{* * *}$ & $41.64^{* * *}$ \\
\hline & 3.27 & 3.20 & 3.09 \\
\hline$N$ & 2223 & 2223 & 2223 \\
\hline$R^{2}$ & 0.306 & 0.238 & 0.205 \\
\hline
\end{tabular}

Robuste Standardfehler in zweiter Zeile; ${ }^{*} p<0.10,{ }^{* *} p<0.05,{ }^{* * *} p<0.01$

Quelle: BIBB-CBS 2007

Wie zuvor gezeigt sinken die unproduktiven Zeitanteile mit zunehmender Ausbildungsdauer. Im Vergleich zu den investitionsorientierten Betrieben sind die Anteile bei den produktionsorientierten Betrieben in allen Jahren signifikant niedriger. Der Koeffizient des unbestimmten Motivs ist im dritten Jahr zwar negativ, aber nicht mehr signifikant. Auffällig ist, dass die Koeffizienten im dritten Jahr meist kleiner und seltener signifikant sind. Dies gilt insbe- sondere für die Großbetriebe, aber auch für einen Großteil der Koeffizienten der Berufsdummies (nicht in Tab. 7 abgebildet). Auch der Anteil an erklärter Varianz ist im dritten Jahr kleiner. Dies spricht dafür, dass die Betriebe vor allem zu Beginn der Ausbildung in ihrer Gestaltungsfreiheit durch die Ausbildungsordnungen und andere berufsund betriebsspezifische Rahmenbedingungen eingeschränkt sind. 
Tab. 8 Zeitaufteilung nach Berufen in Prozent

\begin{tabular}{|c|c|c|c|c|c|c|c|}
\hline & \multicolumn{2}{|c|}{ Anteil unproduktive Zeiten } & \multicolumn{2}{|c|}{ Anteil Fachkräftetätigkeiten } & \multicolumn{2}{|c|}{ Anteil einfache Tätigkeiten } & \multirow[t]{2}{*}{$N$} \\
\hline & Mittelwert & Standardabw. & Mittelwert & Standardabw. & Mittelwert & Standardabw. & \\
\hline \multicolumn{8}{|l|}{ Beruf } \\
\hline Landwirt/in & 26.61 & 16.83 & 31.93 & 12.99 & 41.46 & 17.36 & 40 \\
\hline Gärtner/in & 24.51 & 16.67 & 35.16 & 15.16 & 40.33 & 18.60 & 50 \\
\hline Florist/in & 24.43 & 13.03 & 36.09 & 13.01 & 39.48 & 14.39 & 52 \\
\hline Chemikant/in & 53.97 & 22.16 & 24.37 & 14.98 & 21.66 & 17.16 & 39 \\
\hline $\begin{array}{l}\text { Mediengestalter/in für Digital- und } \\
\text { Printmedien -Mediendesign }\end{array}$ & 30.36 & 20.81 & 43.38 & 22.95 & 26.27 & 16.04 & 35 \\
\hline Drucker/in & 27.27 & 17.83 & 40.04 & 17.65 & 32.69 & 17.86 & 48 \\
\hline Metallbauer/in & 34.56 & 17.83 & 36.98 & 17.11 & 28.46 & 11.42 & 45 \\
\hline $\begin{array}{l}\text { Anlagenmechaniker/in für Sanitär-, } \\
\text { Heizungs- und Klimatechnik }\end{array}$ & 36.66 & 14.45 & 32.64 & 12.59 & 30.70 & 11.61 & 34 \\
\hline Industriemechaniker/in & 52.08 & 22.19 & 25.67 & 14.89 & 22.25 & 15.66 & 63 \\
\hline Kraftfahrzeugmechatroniker/in & 42.77 & 18.11 & 31.51 & 15.71 & 25.72 & 12.08 & 48 \\
\hline Werkzeugmechaniker/in & 47.78 & 22.42 & 30.57 & 17.63 & 21.65 & 13.38 & 63 \\
\hline Zahntechniker/in & 34.99 & 15.53 & 34.85 & 19.28 & 30.15 & 16.56 & 33 \\
\hline Augenoptiker/in & 35.96 & 19.89 & 36.30 & 13.46 & 27.74 & 10.95 & 29 \\
\hline Elektroniker/in für Betriebstechnik & 45.01 & 25.89 & 27.71 & 15.99 & 27.28 & 16.45 & 93 \\
\hline $\begin{array}{l}\text { Elektroniker/in -Energie- und } \\
\text { Gebäudetechnik }\end{array}$ & 37.21 & 15.55 & 31.69 & 15.89 & 31.10 & 12.39 & 41 \\
\hline $\begin{array}{l}\text { Informations- und } \\
\text { Telekommunikationssystem- } \\
\text { Elektroniker/in }\end{array}$ & 31.83 & 15.58 & 32.75 & 16.81 & 35.42 & 14.19 & 48 \\
\hline Mechatroniker/in & 42.81 & 19.82 & 30.91 & 15.70 & 26.28 & 14.08 & 54 \\
\hline Informationselektroniker/in & 34.41 & 15.85 & 35.38 & 15.70 & 30.22 & 11.92 & 39 \\
\hline Bäcker/in & 21.50 & 14.55 & 39.16 & 16.58 & 39.34 & 15.72 & 53 \\
\hline Fleischer/in & 24.46 & 16.46 & 43.37 & 17.03 & 32.17 & 13.44 & 34 \\
\hline Koch/Köchin & 20.37 & 12.50 & 38.02 & 18.36 & 41.62 & 15.12 & 55 \\
\hline Maurer/in & 31.76 & 22.49 & 27.54 & 17.98 & 40.70 & 17.49 & 31 \\
\hline Tischler/in & 36.11 & 18.08 & 27.98 & 16.57 & 35.91 & 19.90 & 47 \\
\hline Maler/in und Lackierer/in & 28.94 & 13.72 & 34.68 & 15.70 & 36.38 & 11.48 & 39 \\
\hline Chemielaborant/in & 38.11 & 18.15 & 40.54 & 20.29 & 21.34 & 18.15 & 30 \\
\hline Bauzeichner/in & 31.00 & 16.24 & 35.88 & 15.89 & 33.12 & 14.13 & 53 \\
\hline $\begin{array}{l}\text { Kaufmann/Kauffrau im Groß- und } \\
\text { Außenhandel }\end{array}$ & 22.48 & 13.60 & 36.23 & 15.76 & 41.29 & 14.38 & 53 \\
\hline Kaufmann/Kauffrau im Einzelhandel & 25.13 & 16.90 & 37.45 & 21.01 & 37.42 & 19.12 & 52 \\
\hline $\begin{array}{l}\text { Fachverkäufer/in im } \\
\text { Nahrungsmittelhandwerk }\end{array}$ & 27.13 & 15.45 & 41.31 & 15.31 & 31.56 & 15.42 & 43 \\
\hline Bankkaufmann/-kauffrau & 36.55 & 16.39 & 30.10 & 16.62 & 33.35 & 14.75 & 32 \\
\hline Versicherungskaufmann/-kauffrau & 37.51 & 19.87 & 35.76 & 17.51 & 26.72 & 16.73 & 47 \\
\hline Reiseverkehrskaufmann/-kauffrau & 28.67 & 17.93 & 40.70 & 17.65 & 30.63 & 12.95 & 44 \\
\hline Veranstaltungskaufmann/-kauffrau & 28.99 & 19.62 & 45.82 & 19.14 & 25.18 & 12.94 & 19 \\
\hline Fachkraft für Lagerlogistik & 28.08 & 21.62 & 32.10 & 19.48 & 39.82 & 21.46 & 54 \\
\hline Steuerfachangestellte/r & 26.10 & 11.07 & 38.78 & 18.67 & 35.13 & 14.60 & 56 \\
\hline $\begin{array}{l}\text { Informations- und } \\
\text { Telekommunikationssystem-Kaufmann/- } \\
\text { kauffrau }\end{array}$ & 33.50 & 18.23 & 34.41 & 15.82 & 32.10 & 13.29 & 58 \\
\hline Informatikkaufmann/-frau & 30.69 & 13.38 & 37.09 & 17.68 & 32.22 & 15.32 & 43 \\
\hline Fachinformatiker/in -Systemintegration & 39.18 & 15.88 & 37.66 & 12.86 & 23.16 & 12.37 & 54 \\
\hline Rechtsanwaltsfachangestellte/r & 23.33 & 19.62 & 41.84 & 20.41 & 34.83 & 19.17 & 44 \\
\hline
\end{tabular}


Tab. 8 (Fortsetzung)

\begin{tabular}{|c|c|c|c|c|c|c|c|}
\hline & \multicolumn{2}{|c|}{ Anteil unproduktive Zeiten } & \multicolumn{2}{|c|}{ Anteil Fachkräftetätigkeiten } & \multicolumn{2}{|c|}{ Anteil einfache Tätigkeiten } & \multirow[t]{2}{*}{$N$} \\
\hline & Mittelwert & Standardabw. & Mittelwert & Standardabw. & Mittelwert & Standardabw. & \\
\hline $\begin{array}{l}\text { Kaufmann/Kauffrau für } \\
\text { Bürokommunikation }\end{array}$ & 26.30 & 18.35 & 35.06 & 16.45 & 38.64 & 12.56 & 44 \\
\hline $\begin{array}{l}\text { Bürokaufmann/Bürokauffrau (Industrie } \\
\text { und Handel) }\end{array}$ & 26.78 & 17.07 & 33.56 & 16.87 & 39.66 & 16.18 & 81 \\
\hline Bürokaufmann/Bürokauffrau (Handwerk) & 27.93 & 9.82 & 35.91 & 10.92 & 36.16 & 12.55 & 31 \\
\hline Industriekaufmann/-kauffrau & 26.22 & 16.31 & 39.17 & 19.12 & 34.61 & 14.63 & 73 \\
\hline (Zahn-)Medizinische/r Fachangestellte/r & 20.55 & 12.52 & 44.48 & 16.70 & 34.97 & 17.51 & 51 \\
\hline Friseur/in & 27.09 & 16.23 & 35.34 & 17.37 & 37.56 & 15.94 & 35 \\
\hline Hotelfachmann/Hotelfachfrau & 22.78 & 15.36 & 44.05 & 18.29 & 33.17 & 16.24 & 56 \\
\hline ÖD-Berufe & 38.95 & 18.08 & 26.00 & 17.42 & 35.05 & 17.00 & 57 \\
\hline
\end{tabular}

Quelle: BIBB-CBS 2007

\section{Zusammenfassung und Schlussfolgerungen}

Dieser Beitrag untersucht auf Basis der BIBB-Erhebung zu Kosten und Nutzen der betrieblichen Ausbildung für das Jahr 2007, ob und wie das Ausbildungsmotiv des Betriebs und die Organisation der Ausbildung zusammenhängen und welche weiteren Faktoren als Erklärung der Ausbildungsorganisation herangezogen werden können. Insbesondere der Einfluss der allgemeinen schulischen Kompetenzen der Auszubildenden, die als Proxy für die Fähigkeiten der Auszubildenden gesehen werden können, wird genauer untersucht. Die Organisation wird dabei anhand der Aufteilung der Zeit der Auszubildenden am Arbeitsplatz abgebildet. In dieser Zeit können die Auszubildenden vom Betrieb für einfache oder Fachkräftetätigkeiten eingesetzt werden und somit produktiv tätig sein oder sie können auf verschiedene Art und Weise „unproduktiv“ ausgebildet werden.

Die Prüfung des Zusammenhangs zwischen dem Ausbildungsmotiv des Betriebs und der Verteilung der unterschiedlichen Ausbildungszeiten zeigt, dass in Betrieben, die ausbilden, um ihren zukünftigen Fachkräftebedarf zu sichern, der Zeitanteil der unproduktiven Tätigkeiten höher ausfällt als in den produktionsorientierten Betrieben. In diesen Betrieben werden sie dafür mit höheren Zeitanteilen sowohl zu einfachen als auch zu Fachkräftetätigkeiten herangezogen. Der häufig geäußerte Vorwurf, dass diese Betriebe die Auszubildenden als „billige Arbeitskräfte“ ausnutzen und sie daher auch nicht an einer qualitativ hochwertigen Ausbildung interessiert sind, kann aber nicht bestätigt werden, da der Unterschied zu den investitionsorientierten Betrieben, die an einer hohen Qualität der Ausbildung interessiert sein müssten, nur relativ gering ist. Mit den vorliegenden Daten kann nicht gemessen werden, zu welchen Unterschieden in der Qualität der Ausbildung die unterschiedli- chen Ausbildungsmotive führen. Es kann also auch nicht pauschal gesagt werden, dass die Ausbildung im Produktionsprozess zu schlechterer Qualität führt als eine Ausbildung, die stärker in Kursen oder einer Lehrwerkstatt durchgeführt wird. In den Betrieben, die nach dem Investitionsmotiv ausbilden, führen die Auszubildenden Fachkräftetätigkeiten aus, um im Produktionsprozess die notwendigen Fähigkeiten zu erlernen, die entstehenden Erträge sind möglicherweise nur von zweitrangiger Bedeutung. Dies könnte eine Erklärung für die nur relativ geringen Unterschiede zwischen den Motiven, insbesondere bei den Fachkräftetätigkeiten, sein.

Zusätzlich werden die Zusammenhänge zwischen der Zeitaufteilung der Auszubildenden und den allgemeinen schulischen Kompetenzen sowie den wirtschaftlichen Rahmenbedingungen untersucht. In einem weiteren Schritt wird dann analysiert, ob sich diese Zusammenhänge nach den Ausbildungsmotiven unterscheiden. Bei den allgemeinen schulischen Kompetenzen kann eine positive Korrelation mit dem Einsatz der Auszubildenden für Fachkräftetätigkeiten festgestellt werden, in den investierenden Betrieben sind dafür die Anteile an unproduktiven Zeiten, in den produktionsorientierten Betrieben die Anteile an einfachen Tätigkeiten niedriger. Nicht geklärt werden kann, ob dies auch innerhalb eines Betriebs gilt, da alle Angaben immer nur im Durchschnitt über alle Auszubildenden des Betriebs getroffen werden. Möglich ist daher auch, dass die Betriebe, die einen stärkeren Einsatz für Fachkräftetätigkeiten anstreben, nur mit Bewerbern mit hohem Niveau an allgemeinen Kompetenzen einen Ausbildungsvertrag schließen. Der hohe Anteil an Fachkräftetätigkeiten wäre also nicht nur Folge der vorhandenen Kompetenzen, sondern vor allem eine strategische Entscheidung des Betriebs. Die Umsetzung dieser strategischen Entscheidung erfordert eine starke Selektion der Bewerber auf Ausbildungsplätze, weil schwächere 
Tab. 9 Deskriptive Statistiken

\begin{tabular}{|c|c|c|c|c|}
\hline & Mittelwert & Standardabw. & Min & Max \\
\hline \multicolumn{5}{|l|}{ Ausbildungsmotiv } \\
\hline Investitionsmotiv & 0.48 & 0.50 & 0 & 1 \\
\hline Unbestimmtes Motiv & 0.29 & 0.46 & 0 & 1 \\
\hline Produktionsmotiv & 0.23 & 0.42 & 0 & 1 \\
\hline \multicolumn{5}{|l|}{ Betriebsgrößenklassen } \\
\hline bis 9 Beschäftigte & 0.33 & 0.47 & 0 & 1 \\
\hline 10 bis 49 Besch. & 0.09 & 0.29 & 0 & 1 \\
\hline 50 bis 499 Besch. & 0.01 & 0.07 & 0 & 1 \\
\hline mehr als 499 Besch. & 0.01 & 0.11 & 0 & 1 \\
\hline \multicolumn{5}{|l|}{ Wirtschaftszweig } \\
\hline Verarbeitendes Gewerbe & 0.36 & 0.48 & 0 & 1 \\
\hline Handel & 0.24 & 0.43 & 0 & 1 \\
\hline $\begin{array}{l}\text { Dienstleistungen Gastgewerbe und } \\
\text { Infrastruktur }\end{array}$ & 0.13 & 0.34 & 0 & 1 \\
\hline Finanzdienstleistungen und Immobilien & 0.15 & 0.35 & 0 & 1 \\
\hline Verwaltung/Erziehung/Gesundheit & 0.12 & 0.33 & 0 & 1 \\
\hline \multicolumn{5}{|l|}{ Region } \\
\hline Ost & 0.16 & 0.36 & 0 & 1 \\
\hline West & 0.84 & 0.36 & 0 & 1 \\
\hline \multicolumn{5}{|l|}{ Betriebstyp } \\
\hline Einzelbetrieb & 0.90 & 0.30 & 0 & 1 \\
\hline Zentrale & 0.04 & 0.20 & 0 & 1 \\
\hline Filiale/Zweigstelle & 0.06 & 0.24 & 0 & 1 \\
\hline Anteil Abiturienten & 0.19 & 0.36 & 0 & 1 \\
\hline allgemeine schulische Kompetenzen & 0.21 & 0.81 & -2 & 2 \\
\hline $\begin{array}{l}\text { Einschätzung der wirtschaftlichen Situation } \\
\text { in Bezug auf Umsatz }\end{array}$ & 0.46 & 0.77 & -2 & 2 \\
\hline $\begin{array}{l}\text { Veränderung der wirtschaftlichen Situation in } \\
\text { den nächsten } 3 \text { Jahren. }\end{array}$ & 0.34 & 0.73 & -2 & 2 \\
\hline Kapazitätsengpässe & 0.36 & 0.48 & 0 & 1 \\
\hline $\begin{array}{l}\text { Anzahl Arbeitskräfte auf dem externen } \\
\text { Arbeitsmarkt }\end{array}$ & -0.15 & 1.14 & -2 & 2 \\
\hline Einsetzbarkeit der externen Fachkräfte & -0.26 & 0.99 & -2 & 2 \\
\hline
\end{tabular}

Quelle: BIBB-CBS 2007

Auszubildende nicht in gleichem Maße produktiv eingesetzt werden könnten. Sollten die Klagen der Betriebe über mangelnde Ausbildungsreife der Schulabgänger berechtigt sein, so hätten sie auf die Kosten-Nutzen-Relation gleich doppelt negativen Einfluss. Zum einen wären zusätzliche Ausgaben für Kurse und Lehrveranstaltungen nötig und zum anderen wäre ein Einsatz für Fachkräftetätigkeiten nur in geringerem Maße möglich.

Insgesamt konnte gezeigt werden, dass der Einsatz der Auszubildenden im Betrieb, neben den Rahmenbedingungen, die z.B. durch den Beruf und die Betriebsgröße vorgegeben werden, mit dem Ausbildungsmotiv des Betriebs und den allgemeinen schulischen Kompetenzen zusammenhängt. Es zeigt sich aber auch, dass die unterschiedlichen Zeitaufteilungen nur teilweise durch die einbezogenen Va- riablen erklärt werden können. Besonders die individuellen Unterschiede zwischen den einzelnen Auszubildenden eines Betriebs können nicht mit einbezogen werden, da hierzu keine Informationen vorliegen. Künftige Forschung zu betrieblichen Ausbildungsmotiven und zur Organisation der betrieblichen Ausbildung sollte die Qualität der Ausbildung explizit mit einbeziehen. Von besonderem Interesse ist der Einfluss des Ausbildungsmotivs auf die Ausbildungsqualität bzw. daraus folgend auf den Erfolg der Ausbildungsabsolventen am Arbeitsmarkt. Dahinter stehen die Fragen, ob das Ausbildungssystem den Ansprüchen des Arbeitsmarktes gerecht wird, den Ausgebildeten tragfähige berufliche Perspektiven eröffnet und den Fachkräftebedarf der Wirtschaft deckt. Sollten Ausbildungsabsolventen aus produktionsorientierten Betrieben im Anschluss an ihre Ausbildung nur 
ausbildungsinadäquate oder gar keine Beschäftigung finden, wäre dies ein Hinweis auf dringenden Handlungsbedarf. Die Analyse des Einflusses der demographischen Herausforderungen auf das Ausbildungsverhalten und die Ausbildungsorganisation der Betriebe sollte Bestandteil zukünftiger Arbeiten sein.

\section{Kurzfassung}

Die duale Ausbildung spielt in Deutschland nach wie vor eine bedeutende Rolle bei der Qualifizierung von Jugendlichen. Etwa $60 \%$ einer Kohorte durchlaufen eine duale Ausbildung. Für die Betriebe entstehen durch die Ausbildung substantielle Kosten, die sie, abhängig von ihrem Ausbildungsmotiv, auf unterschiedliche Weise kompensieren können. Ihre Motivation, warum sie sich an der Ausbildung beteiligen, ist daher ein wichtiger Forschungsgegenstand. Eine Vielzahl an Faktoren kann die Ausbildungsbeteiligung, -motivation und die Ausbildungsorganisation beeinflussen.

Dieser Aufsatz untersucht auf Basis der Erhebung zu Kosten und Nutzen der betrieblichen Ausbildung des Bundesinstituts für Berufsbildung (BIBB) für das Jahr 2007 die Zusammenhänge zwischen dem Ausbildungsmotiv des Ausbildungsbetriebs und den allgemeinen Kompetenzen der Auszubildenden mit der Aufteilung der Arbeitszeit der Auszubildenden im Betrieb.

Unterschieden wird zwischen dem Investitions- und dem Produktionsmotiv. Beim Investitionsmotiv bilden die Betriebe aus, um ihren zukünftigen Fachkräftebedarf decken zu können. Diese Betriebe können Kosten während der Ausbildungsphase in Kauf nehmen, da sie einen Nutzen aus der Weiterbeschäftigung nach der Ausbildung ziehen können. Dieser Nutzen setzt sich aus eingesparten Rekrutierungskosten und der aufgrund von Marktunvollkommenheiten bestehenden Möglichkeit, Löhne unterhalb der Produktivität zahlen zu können, zusammen. Insbesondere eine komprimierte Lohnkurve erhöht den Nutzen von Ausbildungsinvestitionen für den Betrieb. Produktionsorientierte Betriebe nutzen die günstige Arbeitskraft der Auszubildenden, um die Ausbildungskosten decken zu können. In der Regel übernehmen sie ihre Auszubildenden im Anschluss an die Ausbildung nicht.

Die Zuordnung der Motive erfolgt anhand der Übernahmestrategie der Betriebe, die eine generelle, eine teilweise oder keine (bzw. nur in Ausnahmefällen) Weiterbeschäftigung der Auszubildenden nach Abschluss der Ausbildung vorsehen kann. Neben den beiden genannten Motiven wird eine dritte Gruppe von Betrieben gebildet, die keinem der beiden Motive eindeutig zugeordnet werden kann. Dies sind Betriebe, die angeben einen Teil der Auszubildenden übernehmen zu wollen. Nach dieser Operationalisierung folgen
$48 \%$ der Ausbildungsbetriebe dem Investitionsmotiv, $23 \%$ dem Produktionsmotiv und $29 \%$ kann kein Motiv eindeutig zugeordnet werden.

Bei der Zeitaufteilung der Auszubildenden wird zwischen Zeiten, in denen einfache Tätigkeiten ausgeführt werden, Zeiten für Fachkräftetätigkeiten und sonstigen ,unproduktiven" Zeiten, in denen die Auszubildenden auf verschiedene Weise ausgebildet werden, differenziert. Unproduktiv meint dabei, dass unmittelbar keine verwertbaren Produkte oder Dienstleistungen in diesen Zeiten entstehen.

Im Gesamtdurchschnitt verbringen die Auszubildenden $34 \%$ der Zeit im Ausbildungsbetrieb mit einfachen Tätigkeiten, $36 \%$ mit Fachkräftetätigkeiten und in $30 \%$ der Zeit findet unproduktive Ausbildung statt. Multivariate Analysen zeigen, dass der Anteil der unproduktiven Tätigkeiten in den investitionsorientierten Betrieben höher ist als in den Betrieben, die dem Produktionsmotiv zuzuordnen sind. Der Anteil an Fachkräftetätigkeiten ist in den produktionsorientierten Betrieben dafür höher.

Als zweite Hypothese wird getestet, ob die allgemeinen schulischen Kompetenzen der Auszubildenden die Einsetzbarkeit im Betrieb beeinflussen. Als Indikator für die allgemeinen schulischen Kompetenzen wird ein Gesamtwert aus den Angaben der Betriebe über die schriftliche und mündliche Ausdrucksfähigkeit, die Beherrschung der Grundrechenarten, die Grundkenntnisse im IT-Bereich und die Problemlösefähigkeit der Auszubildenden gebildet. Bewertet werden konnte jeweils auf einer Skala von -2 ,sehr schlecht" bis +2 ,sehr gut“. Insbesondere bezüglich der Einsatzzeiten für Fachkräftetätigkeiten kann ein positiver Zusammenhang festgestellt werden. Dies gilt sowohl für die Betriebe mit Investitionsmotiv als auch für die mit Produktionsmotiv. Bei ersteren reduziert sich dafür der Anteil an unproduktiven Tätigkeiten, bei letzteren stärker der Anteil einfacher Tätigkeiten.

Bezüglich verschiedener Betriebseigenschaften zeigt sich, dass in großen Betrieben der unproduktive Anteil deutlich höher ist, dafür fällt insbesondere der Anteil an einfachen Tätigkeiten geringer aus. Auch der Ausbildungsberuf hat einen starken Einfluss auf die Verteilung der Ausbildungszeiten. Zum einen geben die Ausbildungsordnungen die Ausbildungsinhalte vor, zum anderen können die Auszubildenden in manchen Berufen insbesondere zu Beginn der Ausbildung aus Sicherheitsgründen nur beschränkt in den Produktionsprozess integriert werden. Nötige Unterweisungen müssen zunächst in unproduktiven Phasen gelehrt werden.

Insgesamt zeigt sich, dass neben den Betriebscharakteristika das Ausbildungsmotiv und die allgemeinen Kompetenzen der Auszubildenden die Zeitaufteilung im Betrieb beeinflussen. Vor allem der letzte Zusammenhang sollte in zukünftiger Forschung auf individueller Basis Berücksichtigung finden. 


\section{Executive summary: Training Motives and the Allocation of Productive Tasks in Apprenticeship Training in Germany}

The dual system of apprenticeship training still is an important institution concerning the qualification of young adults in Germany. About $60 \%$ of each cohort graduates in an apprenticeship in this system. For firms, apprenticeship training induces substantial costs, which can be compensated in different ways depending on the training motive of the firm. Various factors may have an impact on the training participation of firms, training costs and the organization of the training in the firm.

This paper analyzes the correlation between training motives of firms, the general school competence of apprentices and the allocation of productive tasks at the workplace using data from the cost-benefit-survey of the Federal Institute for Vocational Education and Training for the year 2007 (BIBBCBS 2007). I distinguish between two training motives, the investment and the production motive of training. Investing firms train to safeguard their future need for skilled workers. These firms can save the training costs by retaining apprentices. Benefits of retaining apprentices are the saving of the costs of external recruitments and the rent resulting from the widening gap between productivity and wages due to wage compression. Firms training according to the production motive refinance the training costs by involving the apprentices in the production process. As apprentices' wages are relatively low firms can save wage cost for skilled or unskilled work. These firms usually do not retain their apprentices.

The identification of these motives is via the retention strategy of the firm, which can be: retaining all apprentices, retaining only a part of them or retaining only in exceptional cases. Firms retaining some of the apprentices cannot be assigned to one of the discussed motives, so I include a third group of firms with undefined motive. Using this definition, $48 \%$ of the training firms follow the investment motive, $23 \%$ the production motive and $29 \%$ have an undefined motive.

The time apprentices are available for the firm is divided up into three categories: times of unskilled productive tasks, times of skilled productive tasks and times of "unproductive" tasks. In this case "unproductive" means that, during this time, no usable products or services are produced by the apprentices. On average apprentices conduct $34 \%$ of the time unskilled work, $36 \%$ skilled work and within $30 \%$ of time in the firm they are unproductive. I show that in investment oriented firms, the shares of unproductive tasks are higher than in firms following the production motive.

As a second hypothesis, I test whether there is a correlation between the apprentices' general competences and the allocation of time in the firm. As an indicator for the general competences I use an average value of firms' statements on apprentices' written and oral skills, mathematical skills, problem solving skills, and basic IT-skills. The firms could rate each on a scale from -2 "very bad" up to +2 "very good". As main result I find a positive correlation between general competences and the share of skilled work in firms of both training motives. Investing firms therefore reduce unproductive times while production oriented firms reduce the share of unskilled work.

Additionally, I find that the unproductive share rises with firm size and that vocations have a strong impact on the allocation of time. The latter is caused on the one hand by regulation concerning the curriculum of each training vocation and on the other hand, in some vocations, instructions on safety issues are necessary before involving apprentices in production processes.

As conclusions I find that, beside firm characteristics, the training motive and the general competences have an impact on the allocation of time in apprentices training. Especially the competences should be included in further research on an individual basis.

Danksagung Ich danke Gudrun Schönfeld, Friederike Behringer, Harald Pfeifer, Christian Grund und zwei anonymen Referees für hilfreiche Anregungen und Hinweise.

\section{Literatur}

Acemoglu, D., Pischke, J.-S.: Why do firms train? Theory and evidence. Q. J. Econ. 113(1), 79-119 (1998)

Acemoglu, D., Pischke, J.-S.: Beyond Becker: training in imperfect labour markets. Econ. J. (Lond.) 109(453), F112-F142 (1999a)

Acemoglu, D., Pischke, J.-S.: The structure of wages and investment in general training. J. Polit. Econ. 107(3), 539-572 (1999b)

Bardeleben, R.V., Beicht, U., Fehér, K.: Betriebliche Kosten und Nutzen der Ausbildung. Repräsentative Ergebnisse aus Industrie, Handel und Handwerk. Berichte zur beruflichen Bildung, vol. 187. Bertelsmann, Bielefeld (1995)

Becker, G.S.: Human Capital. A theoretical and Empirical Analysis with Special Reference to Education. Univ. of Chicago Press, Chicago (1964)

Beicht, U., Walden, G., Herget, H.: Kosten und Nutzen der betrieblichen Berufsausbildung in Deutschland. In: Berichte zur beruflichen Bildung. Schriftenreihe des Bundesinstituts für Berufsbildung, vol. 264. Bertelsmann, Bielefeld (2004)

Büchel, F., Neubäumer, R.: Ausbildungsinadäquate Beschäftigung als Folge branchenspezifischer Ausbildungsstrategien. Mitt. Arb.markt- Berufsforsch. 34(3), 269-285 (2001)

Dietrich, H.: Institutional Effects of Apprenticeship Training on Employment Success in Germany (2008). http://www.stanford.edu/ group/scspi/_media/pdf/rc28/conference_2008/p271.pdf (Stand: 29.02.2012)

Dionisius, R., Mühlemann, S., Pfeifer, H., Schönfeld, G., Walden, G., Wenzelmann, F., Wolter, S.C.: Ausbildung aus Produktions- oder Investitionsinteresse? Einschätzungen von Betrieben in Deutschland und der Schweiz. Z. Berufs- Wirtsch.pädag. 105(2), 267-284 (2009a)

Dionisius, R., Mühlemann, S., Pfeifer, H., Walden, G., Wenzelmann, F., Wolter, S.C.: Costs and benefits of apprenticeship training. A comparison of Germany and Switzerland. Appl. Econ. Q. 55(1), 7-37 (2009b) 
Fougère, D., Schwerdt, W.: Are apprentices productive? Appl. Econ. Q. 48(3-4), 317-346 (2002)

Franz, W., Zimmermann, V.: The transition from apprenticeship training to work. Int. J. Manpow. 23(5), 411-425 (2002)

Heinemann, L., Rauner, F.: Qualität und Rentabilität der beruflichen Bildung - Ergebnisse der QEK-Studie im Land Bremen (2008)

Kessler, A.S., Lülfesmann, C.: The theory of human capital revisited: on the interaction of general and specific investments. Econ. J. (Lond.) 116(514), 903-923 (2006) doi:10.1111/j.1468-0297.2006.01116.x

Lindley, R.M.: The demand for apprentice recruits by the engineering industry, 1951-1971. Scott. J. Polit. Econ. 22(1), 1-24 (1975)

Merrilees, W.J.: Alternative models of apprentice recruitment: with special reference to the British engineering industry. Appl. Econ. 15(1), 1-21 (1983)

Mohrenweiser, J., Backes-Gellner, U.: Apprenticeship training: for investment or substitution? Int. J. Manpow. 31(5), 545-562 (2010)

Mohrenweiser, J., Backes-Gellner, U., Zwick, T.: Poaching and firm sponsored training: first clean evidence. Working Paper/Swiss Leading House Economics of Education, vol. 51 (2011)

Mohrenweiser, J., Zwick, T.: Why do firms train apprentices? The net cost puzzle reconsidered. Labour Econ. 16(6), 631-637 (2009)

Pfeifer, H., Schönfeld, G., Wenzelmann, F.: How large is the firmspecific component of German apprenticeship training? Empirical Research in Vocational Education and Training 3(2) (2011)

Pfeifer, H., Wenzelmann, F., Schönfeld, G.: Ausbildungskosten und das Übernahmeverhalten von Betrieben - Ein Vergleich der BIBB-Kosten- und Nutzenerhebungen der Jahre 2000 und 2007. Soz. Fortschr. 59(6-7), 174-182 (2010)

Reglin, T., Schöpf, N. (eds.): Prozessorientierung in der Ausbildung. Ausbildung im Arbeitsprozess. Bertelsmann, Bielefeld (2005)
Sadowski, D.: Berufliche Bildung und betriebliches Bildungsbudget. In: Zur ökonomischen Theorie der Personalbeschaffungs- und Bildungsplanung im Unternehmen. Betriebswirtschaftliche Abhandlungen. Neue Folge, vol. 44. Poeschel, Stuttgart (1980)

Schönfeld, G., Wenzelmann, F., Dionisius, R., Pfeifer, H., Walden, G.: Kosten und Nutzen der dualen Ausbildung aus Sicht der Betriebe. Ergebnisse der vierten BIBB-Kosten-Nutzen-Erhebung. Berichte zur beruflichen Bildung, Bielefeld (2010)

Schweri, J., Mühlemann, S., Pescio, Y., Walther, B., Wolter, S.C., Zürcher, L.: Kosten und Nutzen der Lehrlingsausbildung aus der Sicht Schweizer Betriebe. Beiträge zur Bildungsökonomie, vol. 2. Rüegger, Zürich (2003)

Stevens, M.: An investment model for the supply of training by employers. Econ. J. (Lond.) 104(424), 556-570 (1994)

Wolter, S.C., Ryan, P.: Apprenticeship. In: Hanushek, R., Machin, S., Woessmann, L. (eds.) Handbook of Economics of Education, vol. 34, pp. 208-234. Elsevier, London (2011)

Felix Wenzelmann Studium der Volkswirtschaftslehre an der Rheinischen-Friedrich-Wilhelms-Universität Bonn, 2005 Abschluss als Diplom-Volkswirt, 2006-2007 Wissenschaftlicher Mitarbeiter am Lehrstuhl für Internationale Wirtschaftspolitik, Universität Osnabrück, seit 2007 Wissenschaftlicher Mitarbeiter im Bundesinstitut für Berufsbildung (BIBB) im Arbeitsbereich „Kosten, Nutzen, Finanzierung“.

Forschungsfelder: Berufsbildungsökonomie, insbesondere Kosten und Nutzen betrieblicher Ausbildung und betriebliches Ausbildungsverhalten 